\title{
Temperature forecasting by deep learning methods
}

\author{
Bing Gong ${ }^{1}$, Michael Langguth ${ }^{1}$, Yan $\mathrm{Ji}^{1}$, Amirpasha Mozaffari ${ }^{1}$, Scarlet Stadtler ${ }^{1}$, Karim Mache ${ }^{1}$, and \\ Martin G. Schultz ${ }^{1}$ \\ ${ }^{1}$ Jülich Supercomputing Centre, Forschungszentrum Jülich, 52425 Jülich, Germany
}

Correspondence: b.gong @fz-juelich.de

\begin{abstract}
Numerical weather prediction (NWP) models solve a system of partial differential equations based on physical laws to forecast the future state of the atmosphere. These models are deployed operationally, but they are computationally very expensive. Recently, the potential of deep neural networks to generate bespoken weather forecasts has been explored in a couple of scientific studies inspired by the success of video frame prediction models in computer vision. In this study, a simple recurrent neural network with convolutional filters, called ConvLSTM, and an advanced generative network, the Stochastic Adversarial Video Prediction (SAVP) model, are applied to create hourly forecasts of the $2 \mathrm{~m}$ temperature for the next 12 hours over Europe. We make use of 13 years of data from the ERA5 reanalysis, of which 11 years are utilized for training and one year each is used for validating and testing. We choose the $2 \mathrm{~m}$ temperature, total cloud cover and the $850 \mathrm{hPa}$ temperature as predictors and show that both models attain predictive skill by outperforming persistence forecasts. SAVP is superior to ConvLSTM in terms of several evaluation metrics, confirming previous results from computer vision that larger, more complex networks are better suited to learn complex features and to generate better predictions. The 12-hour forecasts of SAVP attain a mean squared error (MSE) of about $2.3 \mathrm{~K}^{2}$, an anomaly correlation coefficient (ACC) larger than 0.85 , a Structural Similarity Index (SSIM) of around 0.72 and a gradient ratio $\left(r_{G}\right)$ of about 0.82 . The ConvLSTM yields a higher MSE $\left(3.6 \mathrm{~K}^{2}\right)$, a smaller ACC (0.80) and SSIM (0.65), but a slightly larger $r_{G}(0.84)$. The superior performance of SAVP in terms of MSE, ACC and SSIM can be largely attributed to the generator. A sensitivity study shows that a larger weight of the GAN component in the SAVP loss leads to even better preservation of spatial variability at the cost of a somewhat increased MSE $\left(2.5 \mathrm{~K}^{2}\right)$. Including the $850 \mathrm{hPa}$ temperature as additional predictor enhances the forecast quality and the model also benefits from a larger spatial domain. By contrast, adding the total cloud cover as predictor or reducing the amount of training data to eight years has only small effects. Although the temperature forecasts obtained in this way are still less powerful than contemporary NWP models, this study demonstrates that sophisticated deep neural networks may achieve considerable forecast quality beyond the nowcasting range in a purely data-driven way.
\end{abstract}

\section{Introduction}

Accurate predictions of weather are important for many aspects of modern society. They are of high relevance in economy and industry, e.g., for agriculture, for the (renewable) electric power industry or for prevention against natural hazards. Since the early 1960s, numerical weather prediction (NWP) models are run operationally at meteorological centers all over the world. These models are nowadays capable to simulate the dynamics of the global atmosphere down to the kilometre scale (Bauer 
https://doi.org/10.5194/gmd-2021-430

Preprint. Discussion started: 8 March 2022

(c) Author(s) 2022. CC BY 4.0 License.

(c) (i)

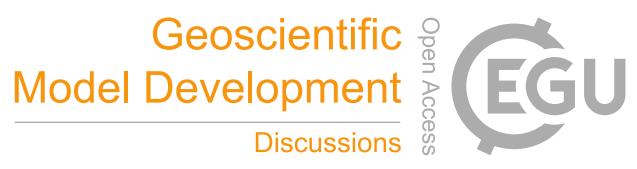

et al., 2015). While their predictions have reached a remarkable degree of reliability, the required computational resources are enormous (Zaengl et al., 2015).

Over recent years, deep learning (DL) has been successfully applied in computer vision applications, such as self-driving cars (Rao and Frtunikj, 2018), human action prediction (Kong and Fu, 2018), and anomaly detection (Liu et al., 2018), etc. These show that deep neural networks have the ability to recognize complex patterns and uncover highly non-linear relations in a data-driven way. Thus, hopes are raised that deep learning can be used for weather prediction and Earth system science (Schultz et al., 2021) which have to deal with many complex, multi-scale and non-linear coupled processes (Orlanski, 1975). The weather and climate communities are beginning to investigate the use of these advanced machine learning methods in the context of weather (McGovern et al., 2017) and climate forecasting (Reichstein et al., 2019), such as data assimilation (e.g. Hatfield et al., 2021), emulation of physical parameterization (e.g. Han et al., 2020), and detection of extreme weather events in climate datasets (e.g. Racah et al., 2016).

As discussed in Schultz et al. (2021), there are many potential applications of DL in the field of weather forecasting. DL methods can be integrated in each step of the NWP workflow which comprises pre-processing of observational data, assimilation of these data into the modelled real atmospheric state, forecasting with a numerical model and post-processing on the raw model outputs (see Fig. 1 in Schultz et al., 2021). Here, we provide a proof-of-concept of replacing the NWP model with data-driven video prediction methods to forecast the evolution of the atmospheric state, particularly the $2 \mathrm{~m}$ temperature up to 12 hours ahead. This is considerably longer than the typical range of nowcasting applications with a lead time of three hours or less (Wilson et al., 2010), but shorter than medium-range forecasts targeted in other studies (c.f. Scher, 2018; Rasp and Lerch, 2018; Weyn et al., 2020). Thus, our application focuses on predicting the diurnal cycle of $2 \mathrm{~m}$ temperature which is potentially challenging for deep neural networks that are known to struggle with periodic processes (Ziyin et al., 2020).

Weather forecasting shares some similarities with video prediction by deep learning. Both explore spatio-temporal patterns from previously observed data to generate a plausible future state of the system. Nevertheless, there are at least two main differences. First, video prediction is mostly used for human pose, physical object and trajectory forecasting, where individual objects are often clearly separable from the background and do not interact with each other on several spatio-temporal scales. Temporal patterns are learned from the movement of objects to then generate a series of frames anticipating how a scene might evolve during the next few seconds. In contrast, weather data does not contain clearly separable objects and the physical laws governing the evolution of weather patterns over time are much more complex due to multi-scale interactions (see, e.g., Orlanski, 1975). For instance, a convective system is driven by large-scale flow patterns (e.g. embedded in a synoptic-scale low) and is subject to turbulence processes in the planetary boundary layer (e.g. convection triggering). Vice versa, the convection itself vents the planetary boundary layer and also modifies the large-scale atmospheric state. Due to the multi-scale interactions, the degree of inherent uncertainty in weather predictions is enormous (e.g. Lorenz, 1969). Second, video predictions mostly aim for perceptually realistic looking scenes. Several evaluation metrics such as the peak signal to noise ratio (Mathieu et al., 2015) or the structural similarity index (Wang et al., 2004) are applied for this purpose in the computer vision domain. However, the degree of physical realism is barely obtainable from a graphical display of weather, for example in weather charts. Due to this difference, meteorologists have developed a broad range of evaluation metrics with careful consideration of their statistical 
https://doi.org/10.5194/gmd-2021-430

Preprint. Discussion started: 8 March 2022

(c) Author(s) 2022. CC BY 4.0 License.

(c) (i)

properties (see, e.g., Wilks, 2011) and deep learning must eventually show that it can compete with numerical models according to the same evaluation standards (e.g. Rasp and Lerch, 2018; Leinonen et al., 2020).

The application of deep neural networks in weather and climate science is still in its infant stage. While some studies experimented with emulators of physical parametrizations within atmospheric models (Brenowitz et al., 2020; Chantry et al., 2021) or processed direct model output for improved forecast products (e.g. Sha et al., 2020; Grönquist et al., 2021), others directly explored video prediction approaches for weather forecasting. So far, relative simple architectures such as fully convolutional u-shaped encoder-decoder networks (U-Net) or convolutional layers coupled with Long Short-Term Memory (LSTM) cells (so called ConvLSTM) are commonly used in the weather forecast domain (e.g. Kim et al., 2017; Weyn et al., 2019; Wang et al., 2021). In parallel, the performance of deep learning models for computer vision tasks has continuously improved with increased complexity and more refined concepts of the neural network architectures. Since the breakthrough of AlexNet (Krizhevsky et al., 2012) in the ImageNet challenge (Deng et al., 2009), convolutional neural networks and their variants have seen rapid development (e.g. Xingjian et al., 2015; Canziani et al., 2016). Recently, Generative Adversarial Networks (GAN; Goodfellow et al., 2014), Variational Auto Encoders (VAE; Kingma and Welling, 2013), and vision transformer networks (Dosovitskiy et al., 2020; Caron et al., 2021) have become increasing popular and are nowadays combined with previous approaches to further improve on machine learning benchmark datasets (see Oprea et al., 2020, for a review).

In this paper, we explore to what extent such more advanced deep learning models with the capability of capturing non-linear relations in the data provide opportunities to enhance the predictive skills of machine learning in Earth Science applications. Accordingly, we have applied a state-of-the-art DL architecture, namely the stochastic adversarial video prediction (SAVP) model which combines ConvLSTM, GAN and VAE architecture components (Lee et al., 2018), to a simplified meteorological forecast problem and compare its results with those from a ConvLSTM model. For convenience, we make use of data from the ERA5 reanalysis system (Hersbach et al., 2020) provided by the European Center for Medium-Range Weather Forecasts (ECMWF). This data has the big advantage of providing a comprehensive estimate on the atmospheric state without suffering from sparse observational data with varying biases due to different measurement techniques (e.g. station sites, radiosondes and satellite observations). Besides, the gridded dataset allows for straightforward applications of convolutional operators. Ultimately, a DL forecast system should work directly on the observational data.

Within the scope of this study, we seek to answer the following research questions: 1) How well do video prediction models perform in predicting the diurnal cycle of $2 \mathrm{~m}$ temperature? 2) Is there a clear advantage of using more sophisticated DL architectures? 3) How do different components in composite model architectures such as SAVP affect the forecast quality? 4) How sensitive is the model performance with respect to external parameters (spatial domain, additional predictors and training dataset size)?

The manuscript is organized as follows: Section 2 will give a thorough review of the state-of-the-art deep learning models for video prediction and also presents some related work on weather forecasting. Section 3 introduces the meteorological dataset and describes the video prediction models that are deployed in this study. In Section 4, a detailed analysis of the model results is presented based on standard evaluation metrics from the domain of computer vision and from the meteorological community. The effect of the different components in SAVP models are analyzed through the sensitivity analysis for the scaling factors on 
https://doi.org/10.5194/gmd-2021-430

Preprint. Discussion started: 8 March 2022

(c) Author(s) 2022. CC BY 4.0 License.

(c) (i)

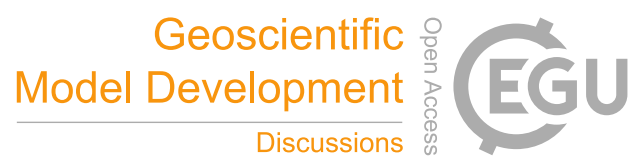

L1-loss. We also present the results of sensitivity analysis to evaluate the impacts of input variable selection, the size of the spatial domain, and the length of the training dataset. Finally, Section 6 summarizes the findings and provides an outlook on the future avenue of weather forecasting with video prediction methods.

\section{State-of-the-art video prediction models}

\subsection{Deep learning for video prediction}

Common machine learning techniques for video prediction can be categorized into Recurrent Neural Networks (RNN) (Oliu et al., 2018; Wang et al., 2018), Adversarial Learning (Goodfellow et al., 2014; Mathieu et al., 2015), and VAE (Patraucean et al., 2015). While different recurrent network architectures have been developed over the last years, LSTM cells combined with convolutional layers as proposed by Xingjian et al. (2015) have been widely applied for video prediction as baseline model to compare with other state-of-the-art methods (Villegas et al., 2017; Guen and Thome, 2020). The combination of convolution with LSTM enables the DL model to capture spatio-temporal dependencies and thus make predictions about the temporal evolution of spatial patterns, which is the core task of video prediction.

Despite the early success of ConvLSTM models, they are prone to generate blurry images, which don't look very realistic (Denton and Fergus, 2018; Ebert et al., 2017). The reason for this can be attributed to the loss function used in ConvLSTM models where the L1- and L2-loss constitute common choices. These losses measure the point- or pixel-wise distance between prediction and ground truth and rely on the assumption that the data follows a Gaussian distribution. However, L1- and L2losses perform poorly when the data is drawn from multi-modal distributions or from non-Gaussian distributions. The problem gets worse with growing uncertainty of the future state (Mathieu et al., 2015). This is because the model tends to converge towards the average of all the possible future states on a point-wise level even if the average values themselves have low probability. This failure in capturing and reflecting the statistical nature of the underlying data leads to a rather quick degradation of forecast accuracy with increasing lead times as noted by Mathieu et al. (2015) and Sun et al. (2019).

As an alternative, GAN-based architectures have been developed, which use adversarial loss to learn the underlying data statistics among multiple equally probable modes and therefore mitigate blurriness. GANs constitute a composite model architecture which consists of a generator and a discriminator model. The discriminator is trained to distinguish between real and artificially generated video sequences. Conversely, the generator gets optimized to fool the discriminator, i.e. it aims to produce video sequences that cannot be differentiated from real ones by the discriminator. By training both models adversarially, the generator must learn the statistical properties of the underlying data and thereby becomes capable to generate perceptually realistic images (Oprea et al., 2020).

However, GAN models have also their shortcomings. It is well known that these models may lack diversity in the predicted video sequences which is commonly referred as mode collapse in the computer vision community (Isola et al., 2017; Lee et al., 2018). Approaches to overcome model collapse are either to optimize on the Earth Mover distance (Wasserstein GANs) instead of the cross-entropy loss (Gulrajani et al., 2017) or to embed a VAE framework (Kingma and Welling, 2013). VAEs, like other 
https://doi.org/10.5194/gmd-2021-430

Preprint. Discussion started: 8 March 2022

(c) Author(s) 2022. CC BY 4.0 License.

(c) (i)

likelihood-based models, can play a complementary role to the GANs and generate more dispersed samples, better learn the data distribution and avoid mode-collapse.

To leverage the advantages of different architectures, Lee et al. (2018) proposed a model architecture that tries to overcome the aforementioned shortcomings by combining three different model architectures. Their SAVP approach incorporates VAE and GAN components together with ConvLSTM cells. Since SAVP leverages the advantages of both, this model demonstrates very good forecasting capability when applied to common ML benchmark datasets such as Moving-MNIST, BAIR Push and KTH (Franceschi et al., 2020; Jin et al., 2020).

\subsection{Video prediction in weather forecasts}

Precipitation nowcasting with a lead time of up to three hours is one of the most common application of video prediction models. Lagrangian persistence approaches with optical flow (Reyniers, 2008; Ayzel et al., 2019) are well established and already outperform NWP models. The limited performance of NWP models for such short-term forecasts is related to spinup effects after initialization and to the data assimilation procedure, which is challenged by quickly varying atmospheric processes with non-Gaussian statistical properties such as cloud formation and precipitation. However, optical flow methods fail to capture any developments in the precipitation patterns and thus, advanced deep neural network architectures have been recently applied to attain further improvements. Corner stones in the history of precipitation nowcasting are the study by Xingjian et al. (2015) and the development of PredRNN (Wang et al., 2017) who both applied ConvLSTM models for this task. Recently, different model architectures with increasing complexity have been tested such as attention models (e.g., Sønderby et al., 2020) and deep U-Nets (e.g Ayzel et al., 2020). Recently, GAN-based models are becoming popular for precipitation nowcasting since they succeed in preserving the underlying statistical distribution and thereby improve in forecasting stronger precipitation events (Liu and Lee, 2020; Ravuri et al., 2021).

For longer lead times, NWP models still constitute the state-of-the-art (Bauer et al., 2015; Schultz et al., 2021), but there have been a few experimental studies which examine the applicability of deep neural networks to generate tailor-made meteorological predictions in the short, medium and seasonal forecast range (more than 6 hours, up to two weeks and beyond).

Weyn et al. (2019) developed weather prediction models using deep convolutional neural networks (CNNs) to predict the 500-hPa geopotential height at a lead time of 14 days. Rasp and Thuerey (2021) proposed a deep residual convolutional neural network (ResNet) to predict global geopotential, temperature and precipitation at $5.625^{\circ}$ resolution up to 5 days ahead based on the WeatherBench dataset (Rasp et al., 2020). The study from Weyn et al. (2020) explored a CNN-based model to predict surface temperature patterns. These results and other studies such as Scher (2018); Chattopadhyay et al. (2020) show that basic meteorological features (e.g. evolving Rossby waves) can be predicted from DL models and that a realistic seasonal cycle with prescribed variations in top-of-atmosphere solar forcing can be produced. Even though the DL models still cannot compete with operational NWP models on high spatial resolution, these first results are promising. One aspect which makes DL models particularly attractive is that they are computationally cheap once the neural network has been trained.

However, despite of these initial successes, we observe that DL models for weather forecasting optimized on the L1- and L2-loss also suffer from a similar issue as generating "blurry images" in computer vision tasks. Distinct meteorological fea- 
tures such as precipitation patterns or weather fronts often get smoothed and thus, the predicted meteorological fields exhibit statistical properties that do not match the observed ones.

To improve handling of the inherent uncertainty and to preserve the high spatio-temporal variability in meteorological forecast products, Bihlo (2020) trained conditional GAN (cGAN) models based on the pix2pix architecture (Isola et al., 2017) with a U-net deployed for the generator. With this architecture, he predicted the $500 \mathrm{hPa}$ geopotential height, the $2 \mathrm{~m}$ temperature and total precipitation for a maximum lead time of 24 hours and attained encouraging results for the two former quantities. Similar to our study, they used ERA5 reanalysis data sliced to a region over Europe and attained promising results on a coarsened $0.5^{\circ}$ grid.

Our study builds on these recent works by employing the SAVP model architecture to weather forecasts over 12 hours. As described above, SAVP combines the advantages of GANs with those from VAE, and we can thus hope to obtain accurate predictions with sharp features. We compare the SAVP results to a simple ConvLSTM model to probe the sensitivity of the forecast quality on the complexity of the model architecture. Furthermore, we examine the impact of the target domain size, the number of selected predictors and the size of the training dataset.

\section{Data and Models}

\subsection{Dataset}

The ERA5 reanalysis dataset provided by the ECMWF is used as the data source in this study (Hersbach et al., 2020). Reanalysis data combines a numerical weather prediction (NWP) model, in this case the Integrated Forecast System (IFS) Cy41r2, with sophisticated data assimilation to retrieve an optimized estimate on the atmospheric state. Global atmospheric reanalysis datasets such as the ERA5 play a substantial role in climate monitoring and are also used over a wide range of other applications in Earth Science, e.g. for hydrological studies (e.g. Tarek et al., 2020) or to track progress in numerical modelling (e.g. Haiden et al., 2021).

The original ERA5 reanalysis data is defined on a reduced N320 Gaussian grid with an approximate horizontal grid spacing of $0.2825^{\circ}(\Delta x \approx 30 \mathrm{~km})$. Since such highly resolved data fields with 640 grid points in latitude direction and about 1280 grid points in longitude direction near the equator would consume too much memory for the video prediction task, we limit our forecasting task to the region of Central Europe (see Fig. 1) and subset the data accordingly.

The deep learning task of our study is to generate hourly forecasts of the $2 \mathrm{~m}$ temperature over the next 12 hours based on the ERA5 reanalysis fields of the previous 12 hours. The two neural networks used are described in Section 3.3. Since the near surface temperature is the product of complex physical processes between the surface, the planetary boundary layer and the free troposphere, we additionally exploit other meteorological variables from the ERA5 dataset. In particular, we use the $850 \mathrm{hPa}$ temperature $\left(T_{850 \mathrm{hPa}}\right)$ and the total cloud cover $(\mathrm{TCC})$ as additional informative predictors. $T_{850 \mathrm{hPa}}$ corresponds to the air temperature at a height of approximately $1500 \mathrm{~m}$ above sea level and is commonly used to characterize air masses since it is not directly affected by processes at the surface. This variable has been used in previous $2 \mathrm{~m}$ temperature forecasting studies for statistical post-processing of surface air temperatures by machine learning methods (Casaioli et al., 2003; Eccel et al., 2007). 
TCC is supposedly relevant since clouds impact the incoming solar radiation at daytime and thus modulate the cooling of the surface and the atmosphere. Thus, both variables are assumed to encode information on the atmospheric state that may help a data-driven model to predict the evolution of the $2 \mathrm{~m}$ temperature on a sub-daily scale. The choice of the predictor variables has been made based on expert knowledge. A more systematic variable selection process as is typical for data science studies is beyond the scope of this paper.

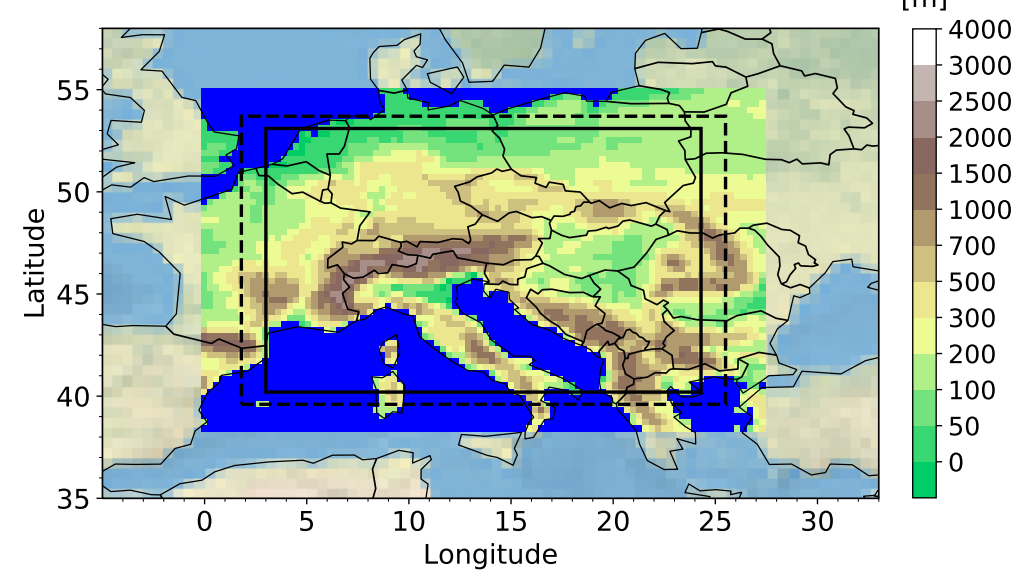

Figure 1. Topographic height of the surface from the ERA5 reanalysis dataset remapped onto a regular, spherical grid with $\Delta x=0.3^{\circ}$. The grid boxes of the target domain are highlighted by opaque colours. The dashed and solid lines bound the subdomains in the sensitivity study. The domains comprise $92 \times 56,80 \times 48$ and $72 \times 44$ grid points in zonal and meridional direction, respectively.

\subsection{Preprocessing}

To allow the application of convolutional operations, the data was interpolated onto a regular spherical grid with a spacing of $0.3^{\circ}$ via the Meteorological Archival and Retrieval System (MARS) in this study. On this grid, the target domain over Europe consists of $92 \times 56$ grid points in zonal and meridional direction, respectively. Finally, we restrict the time period to the years 2007-2019 (13 years). In this way, no large climate change signals are involved in the temperature field. The data was originally recorded hourly and further processed as samples for training deep learning network. Each sample consists of a 24 time steps, of which 12 is used as input for the next 12 hours forecasts. This results into about 8400 samples per year.

We constructed three cross-validation experiments by selecting different non-overlapping data splits for training, validation and testing (see Fig. 2). Thereby, we make use of 11 years of data for training, while two years are deployed for validation and testing with minimized auto-correlation following the discussion in Schultz et al. (2021). The cross-validation is applied to check the robustness of the trained models over a broad temperature range. By selecting 2010 (CV 3), 2016 (CV 1) and 2019 (CV 2) for the testing dataset, we ensure that our trained models are tested on years with relatively cool, on-average and warm 
temperatures, respectively, within the chosen data period. During training, the validation loss (for tuning the model parameters) operates on data from 2013 (average), 2017 (warm) and 2016 (average).

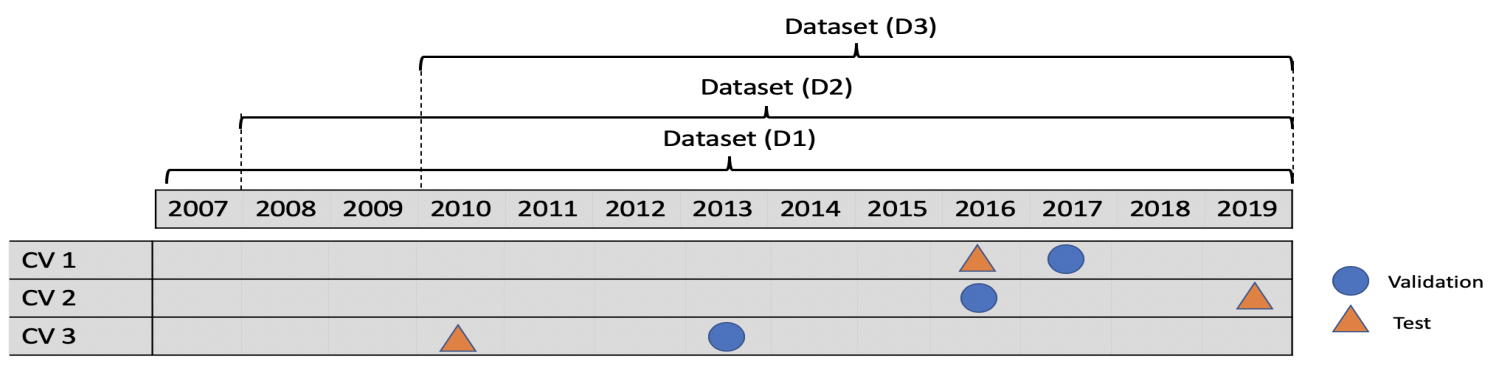

Figure 2. The three cross-validation datasets (CV1, CV2, and CV3) consist of different training, validation and testing samples from the 13 years ERA5 reanalysis data between 2007 and 2019. Each row represents one data partition. The three datasets (D1, D2, and D3) consist of samples from 2007 to 2019, from 2008 to 2019, and from 2010 to 2019, respectively.

To check how the model performance depends on the spatial domain size and on the provided information of the atmospheric state, we vary the spatial extent of the domain and the number of involved predictors. For the former, smaller target regions are tested, see Fig. 1. The latter is realized by first removing TCC from the list of predictors in a first and just inputting $T_{2 \mathrm{~m}}$ in a second experiment. Additionally, we vary the number of training samples by cropping the training dataset. A comprehensive overview on the sensitivity experiments is provided in Table A1 of the Appendix.

Our approach shares some similarities with the study of Bihlo (2020) as we use the same dataset, a similar study region and also focus on short range predictions. However, besides the use of a different neural network architecture (SAVP in our case, cGAN in Bihlo (2020)), there are some distinct differences which make our application potentially more challenging. First, we approximately retain the spatial resolution of the ERA5 reanalysis data on a regular $0.3^{\circ}$ grid (compared to $0.5^{\circ}$ ). Besides, the temporal resolution is also considerably higher since we set the time step to one hour compared to three hours. By doing so, our models must learn to represent smaller features of the near surface temperature field and they must better capture the underlying diurnal cycle. By conditioning on the own predictions, errors are expected to accumulate quicker with an hourly time step.

\subsection{Model architectures}

In the following, we briefly introduce the two video prediction models probed in this study, the convolutional LSTM (ConvLSTM) model and the Stochastic Adversarial Video Prediction (SAVP) model. While a summary on both architectures is provided, more detailed descriptions can be obtained from the original papers, that is the study by Xingjian et al. (2015) on ConvLSTM networks and the work by Lee et al. (2018) for SAVP. 
https://doi.org/10.5194/gmd-2021-430

Preprint. Discussion started: 8 March 2022

(c) Author(s) 2022. CC BY 4.0 License.

(c) (i)

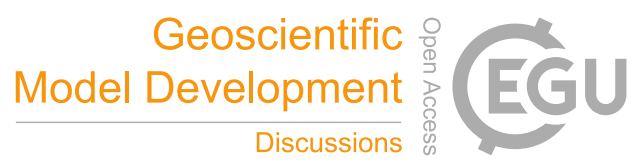

\subsubsection{The convolutional LSTM (ConvLSTM) model}

The ConvLSTM model employs convolutional operations which encodes the spatial properties of the input data into a hidden state. Temporal coherence is preserved with the help of a gated LSTM, so that an encoded state from all input data is achieved at the end of the input sequence (the encoded atmospheric state over the previous 12 hours in our case). The forecasting network then unfolds this state by conveying both, the LSTM cells' states (cell and hidden state) and the predictions fed in sequentially to come up with a forecast over the next 12 hours. Here, we employ a one-layer ConvLSTM network, i.e. one ConvLSTM layer followed by a $1 \times 1$ convolutional layer. A sketch of the model architecture is provided in Fig. 3. We used a batch size of 4 and applied the Adam optimizer (Kingma and Ba, 2014) with a learning rate of $l_{r}=0.001$ to train the model for 10 epochs.

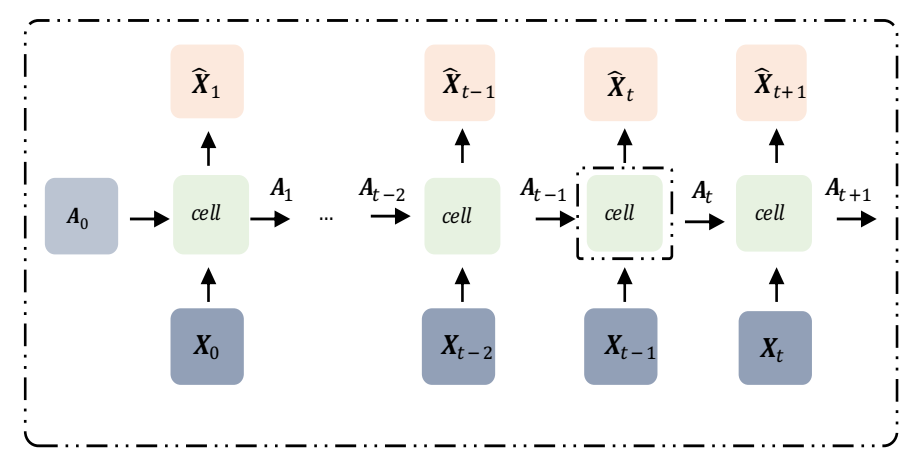

(a) LSTM Future Predictor Model

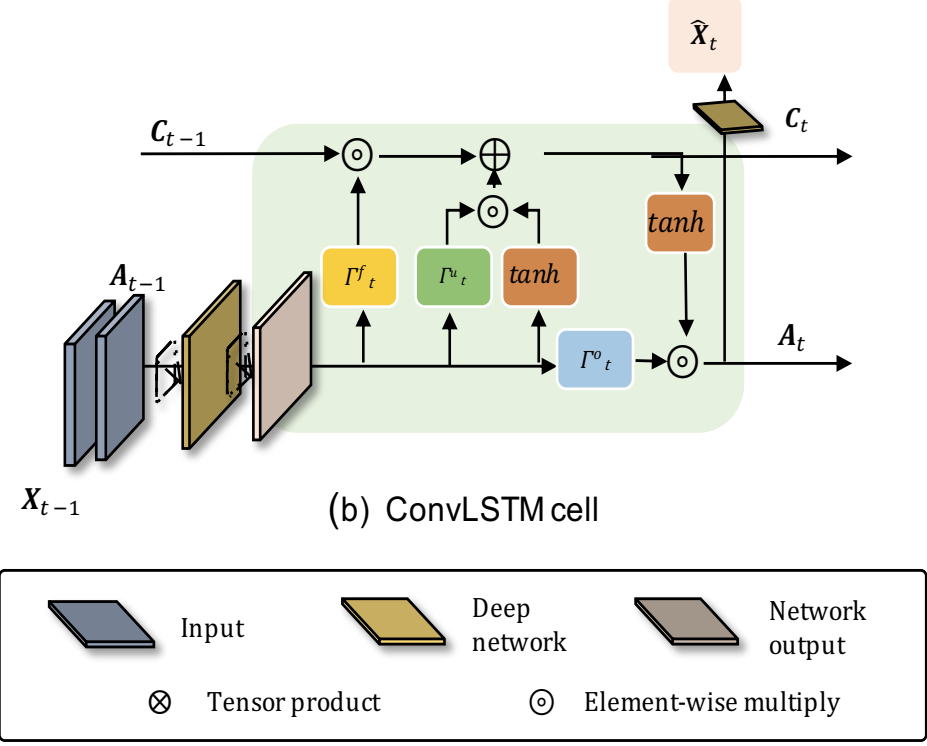

Figure 3. Sketch of ConvLSTM architecture (Xingjian et al., 2015). 
https://doi.org/10.5194/gmd-2021-430

Preprint. Discussion started: 8 March 2022

(c) Author(s) 2022. CC BY 4.0 License.

(c) (1)

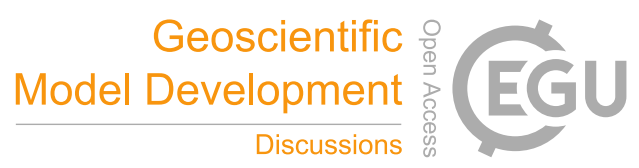

\subsubsection{The Stochastic Adversarial Video Prediction (SAVP) model}

SAVP constitutes a combination of the GAN and the VAE architectures. The model is therefore best described by summarizing both components separately in the following subsections before we explain how these components are coupled together. The explanations are furthermore complemented by a sketch of the SAVP architecture provided in Fig. 4.

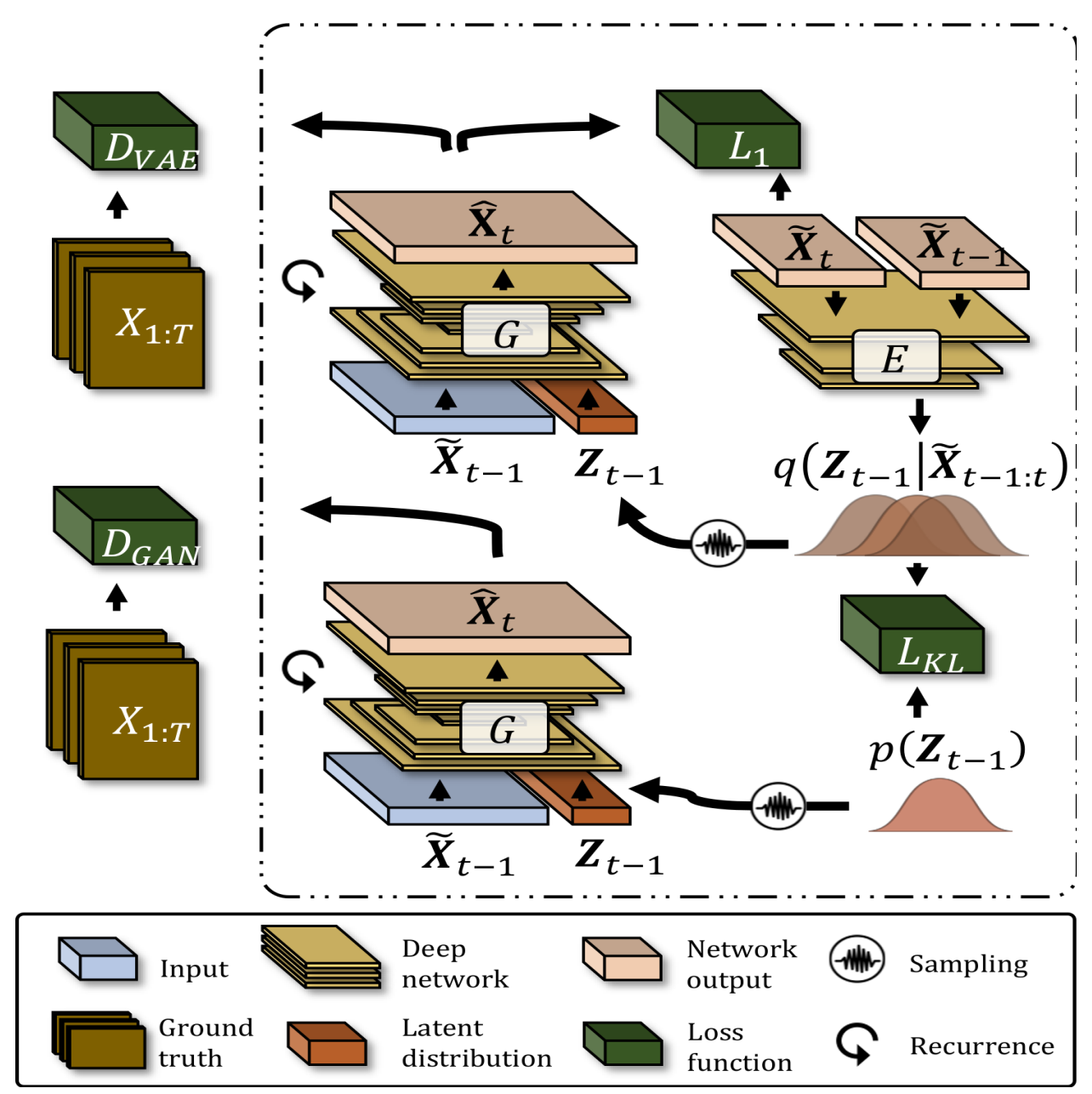

Figure 4. Illustration of the SAVP architecture (from Lee et al., 2018).

Variational autoencoder: The VAE part of SAVP consists of an encoder and a generator. The deep encoder $E$ compresses the information from adjacent time steps into a low-dimensional latent vector $\mathbf{Z}_{t-1}=E\left(\tilde{\mathbf{X}}_{t}, \tilde{\mathbf{X}}_{t-1}\right)$ where $\tilde{\mathbf{X}}$ represents either ground data from the input sequence or previously predicted data (i.e. $\tilde{\mathbf{X}}_{t} \in\left\{\mathbf{X}_{t}, \hat{\mathbf{X}}_{t}\right\}$ ). Thus, $E$ encodes the transition between the states at time steps $t-1$ and $t$ into the latent representation $\mathbf{Z}_{t-1}$ which is then fed to the deep generator $G$ for reconstructing the state $\hat{\mathbf{X}}_{t}$. To control the latent space and to allow random sampling, the learned posterior distribution $q\left(\mathbf{Z}_{t-1} \mid \tilde{\mathbf{X}}_{t-1: t}\right)$ is kept close to a prior distribution $p\left(\mathbf{Z}_{t-1}\right)$. Thus, the loss component of the VAE part consists of the $L_{1}$-error constituting the 
reconstruction loss (first term in Eq. 1) and the Kullback-Leibler divergence $D_{K L}\left(q\left(\mathbf{Z}_{t-1} \mid \tilde{\mathbf{X}}_{t-1: t}\right) \| p\left(\mathbf{Z}_{t-1}\right)\right)$ which acts to regularize the posterior distribution of the latent space onto the prior distribution (second term in Eq. 1). The latter constitutes a standard Gaussian distribution whose parameters are optimized with the help of the re-parametrization trick (Kingma and Welling, 2013).

Generative adversarial network: The generator $G$ in SAVP inputs the data from the previous time step $\tilde{\mathbf{X}}_{t-1}$, i.e. either ground truth or previously generated data, to reconstruct the data at time step $t$. Additionally, the generator is also condinoned on the latent space $t_{-1}$ via sampling. In GAN-based networks the generator $G$ is thereby encouraged to learn the statistical properties of the underlying data. This is achieved by optimizing $G$ to fool a deep discriminator $D$ which is itself optimized to distinguish between real data (i.e. the ground truth) and generated data. The loss function of the GAN $\mathcal{L}_{G A N}$ in SAVP applies the binary cross-entropy loss for an adversarial minimax-optimization. While the generator tries to minimize $\mathcal{L}_{G A N}$, the discriminator aims to maximize $\mathcal{L}_{G A N}$.

Combining VAE and GAN: For the SAVP architecture, one generator $G$ is set-up which is shared between the VAE and GAN parts. However, two separate discriminators are used which are equivalent in terms of the architecture, but differ in their trainable model parameters. The latter difference arises from the latent embedding that is fed to the shared generator $G$. For the discriminator related to the VAE part $D^{V A E}, \mathbf{Z}_{t-1}$ is sampled from the posterior distribution $q\left(\mathbf{Z}_{t-1} \mid \tilde{\mathbf{X}}_{t-1: t}\right)$, whereas sampling from the prior distribution $p\left(\mathbf{Z}_{t-1}\right)$ is performed for the discriminator of the GAN part. Consequently, two GAN-loss terms become part of SAVP's objective function (see Eq. 1) whose third and fourth term are related to the GAN and VAE component, respectively. The total SAVP loss is calculated as:

$$
G^{*}, E^{*}=\arg \min _{G, E} \max _{D^{V} D^{V A E}} \lambda_{1} \mathcal{L}_{1}(G, E)+\lambda_{K L} \mathcal{L}_{K L}(E)+\mathcal{L}_{G A N}(G, D)+\mathcal{L}_{G A N}^{V A E}\left(G, E, D^{V A E}\right) .
$$

The discriminator architecture used by SAVP involves several convolutional layers that operate on the complete data sequence, followed by a fully connected layer. 3D convolutions are applied to handle the spatial and the temporal dimensions simultaneously. The shared generator $G$ involves several ConvLSTM-layers with internal skip connections. These are followed by two convolutional layers to predict the state at the next time step. A separate composite mask is used to identify features from the data that are displaced between the time steps (e.g. a cold-front in our meteorological application). In total, the number of trainable model parameters sums up to about 14M. More details on the architecture are provided in Lee et al. (2018).

The default hyper parameters and training schedule in this study have been modified from the original description in Lee et al. (2018). During training, the initial learning rate used with the Adam optimizer is set to $l_{r}=2 \times 10^{-4}$. The training applies linear learning rate decay to $l_{r}=2 \times 10^{-8}$ between the iteration steps 3000 to 9000 . With a batch size of 32 , this roughly corresponds to the start of the second epoch and the end of the third epoch, respectively. Overall, the model is optimized on four epochs. For the scaling factors of the different loss components, we choose $\lambda_{1}=10000$ and $\lambda_{K L}=0.01$. Note that variations in $\lambda_{1}$ attain special attention in our sensitivity analysis below. The scaling factor for the distance in features space is setup to 0.001 . Furthermore, the reconstruction loss only accounts for $T_{2 \mathrm{~m}}$ which differs from common computer vision applications where all channels enter this loss term. 


\subsection{Evaluation metrics}

For evaluating the video frame prediction models introduced above, we make use of metrics that are commonly applied in the meteorological domain as suggested by Rasp and Thuerey (2021). In particular, we calculate the mean square error (MSE) and the anomaly correlation coefficient (ACC) for the predicted $T_{2 \mathrm{~m}}$-fields (cf. Appendix Eq. B1 and Eq. B2). While the MSE measures the mean squared distance between the predicted and the analyzed (ground truth) temperature field, the ACC quantifies the agreement in the spatial patterns of departures from the climatological mean. Thus, the ACC is a positively oriented score with a perfect value of 1 , whereas the MSE is negatively oriented with a perfect value of 0 . In this study, we make use of the uncentered ACC (see Eq. B2) and calculate the climatological mean based on 30 years of data (1991-2020) provided by the ERA5 dataset. The climatological mean is computed for each month of the year and each hour of the day separately. This ensures that the seasonal and diurnal cycle of the near-surface temperature are incorporated.

In addition to the meteorological evaluation metrics, we also choose the Structural Similarity Index (SSIM) which is commonly applied in video prediction to access the perceptual similarity between images (Wang et al., 2004). Transferring to this application, the SSIM quantifies and compares the mean as well as the spatial variability in the predicted $2 \mathrm{~m}$ temperature field against the ground truth and also accounts for covariances (cf. Eq. B7). Although being an evaluation metric from computer vision, it is considered to provide useful information on the forecast quality. Like the ACC, the SSIM is a positively oriented score with a perfect value of 1 .

To evaluate the truthfulness of the predicted spatial variability, we also compute the domain-averaged amplitude of the horizontal $T_{2 \mathrm{~m}}$-gradient. Similar to Sha et al. (2020), we then calculate the ratio $r_{G}$ of the gradient amplitude from the predictions and the respective ground truth (see Eq. B4). For $r_{G}(\mathbf{X}, \hat{\mathbf{X}})=1$, the predictions share the same local spatial variability as the ERA5 reanalysis data, while $r_{G}(\mathbf{X}, \hat{\mathbf{X}})<1\left(r_{G}(\mathbf{X}, \hat{\mathbf{X}})>1\right)$ indicates that the local spatial variability is underestimated (overestimated) in the predictions. This metric is similar to the sharpness measure introduced in Mathieu et al. (2015), but takes the Earth's curvature into account and does not scale to the maximal gradient amplitude.

While further details on all evaluation metrics are provided in Appendix C, we verify our models against the persistence forecast in terms of skill scores for convenience. This allows a direct comparison with a reference model which attains a value of $S_{r e f}$ for the respective score $S$. As reference model, we use a simple persistence model which is based on the assumption that today's weather is the same as yesterday's, i.e. the temperature field from the last day is simply copied. Together with the perfect score value $S_{p e r}$, the skill score $S S_{S}$ reads

$$
S S_{S}=\frac{S_{m}-S_{r e f}}{S_{p e r}-S_{r e f}}
$$

where $S_{m}$ denotes the score value of the considered model forecast.

\section{Results}

315 In the following, we evaluate the predictive skill of the SAVP and ConvLSTM models for $2 \mathrm{~m}$ temperature predictions up to a lead time of 12 hours. For the presented model results, our default hyper parameters of both models have been tuned to yield 
https://doi.org/10.5194/gmd-2021-430

Preprint. Discussion started: 8 March 2022

(c) Author(s) 2022. CC BY 4.0 License.

\section{(c) (i)}

best results in terms of the MSE. However, we also provide an ablation study on the L1-loss component in the SAVP model and also probe the sensitivity with respect to input variables, regions and size of training dataset.

\subsection{Model performance analysis}

The skill scores in terms of the MSE (a), the ACC (b) and the SSIM (c) are displayed in Fig. 5. The uncertainty estimates depicted by the boxes and whiskers are derived through block bootstrapping with a block length of seven days (Efron and Tibshirani, 1994). It is seen that all video prediction models outperform the persistence forecast significantly over the the complete prediction period. The constant MSE of the persistence forecast (MSE(Persistence) $\simeq 7 \mathrm{~K}^{2}$ ) is reduced by about $50 \%$ for ConvLSTM and about $70 \%$ for SAVP model over the prediction period. Likewise, both models also clearly provide better forecasts of local temperature anomalies from the climatological mean $(A C C($ Per sistence $) \simeq 0.67)$ as seen from Fig. 5 . Only with respect to the SSIM $($ SSIM $($ Persistence $) \simeq 0.66)$, the ConvLSTM model loses its forecast skill after a lead time of seven hours (Fig. 5c). In many aspects, the SAVP models also performs clearly better than the ConvLSTM model: The MSE skill score degrades linearly and at a much smaller rate, especially over the first six hours. After 12 hours, the MSE of SAVP tracks at about $2.3 \mathrm{~K}^{2}$, while the ConvLSTM-model shows up with a MSE slightly above $3.6 \mathrm{~K}^{2}$ (absolute score values are displayed in Fig. A1 of the Appendix). Similarly, the ACC and the SSIM remain closer to 1 for the SAVP model $(A C C(S A V P) \simeq$ $0.87>A C C($ ConvLSTM $) \simeq 0.80$ and $\operatorname{SSIM}(S A V P) \simeq 0.73>\operatorname{SSIM}(\operatorname{ConvLSTM}) \simeq 0.67)$. Thus, as expected, the more complex SAVP model can learn a better representation of the atmospheric state and therefore produces a better prediction of the diurnal cycle of the $2 \mathrm{~m}$ temperature compared to ConvLSTM.

However, even though the global variability as expressed by the SSIM is better captured with SAVP, the local spatial variability is scarcely better than in the ConvLSTM model. In terms of the horizontal gradient ratio, the forecasts of both model degrade continuously with increasing lead time yielding a noticeably underestimation by the end of the forecast period (see Fig. 5d). Thus, the predicted $2 \mathrm{~m}$ temperature fields of both video prediction models become too smooth indicating that small-scale variations due to the underlying topography (mountain ranges) and surface type (costal regions) get blurred.

To illustrate concretely our statistical findings, we show a representative case study of ConvLSTM and SAVP forecasts starting on 2019-08-02, 03:00 UTC. The first row in Fig. 6 shows the $2 \mathrm{~m}$ temperature field from the ERA5 reanalysis (ground truth) for a lead time of six and 12 hours. The differences of the respective ConvLSTM and SAVP model forecasts are presented in the second and third row, respectively, with positive values corresponding to a warm bias.

Apart from growing differences to the ground truth with increasing lead time where SAVP exhibits smaller errors on average, both models forecast strong warming over continental areas. Thus, aspects of the diurnal cycle are captured by the video prediction models. However, it is also noted that the forecast accuracy especially deteriorates around the Alpine region, indicating that both models have problems in predicting the temperature evolution in this area. Besides, differences appear to be bounded by the coastal line with dipole structures visible in the Mediterranean region. This indicates that strong spatial gradients in $2 \mathrm{~m}$ temperature tend to be blurred in accordance with the findings in Fig. 5d.

Further insight into the statistical properties of the forecast with respect to the ERA5 reanalysis (the ground truth) can be obtained from conditional quantile plots. These plots visualize important aspects of the joint distribution of forecast and 

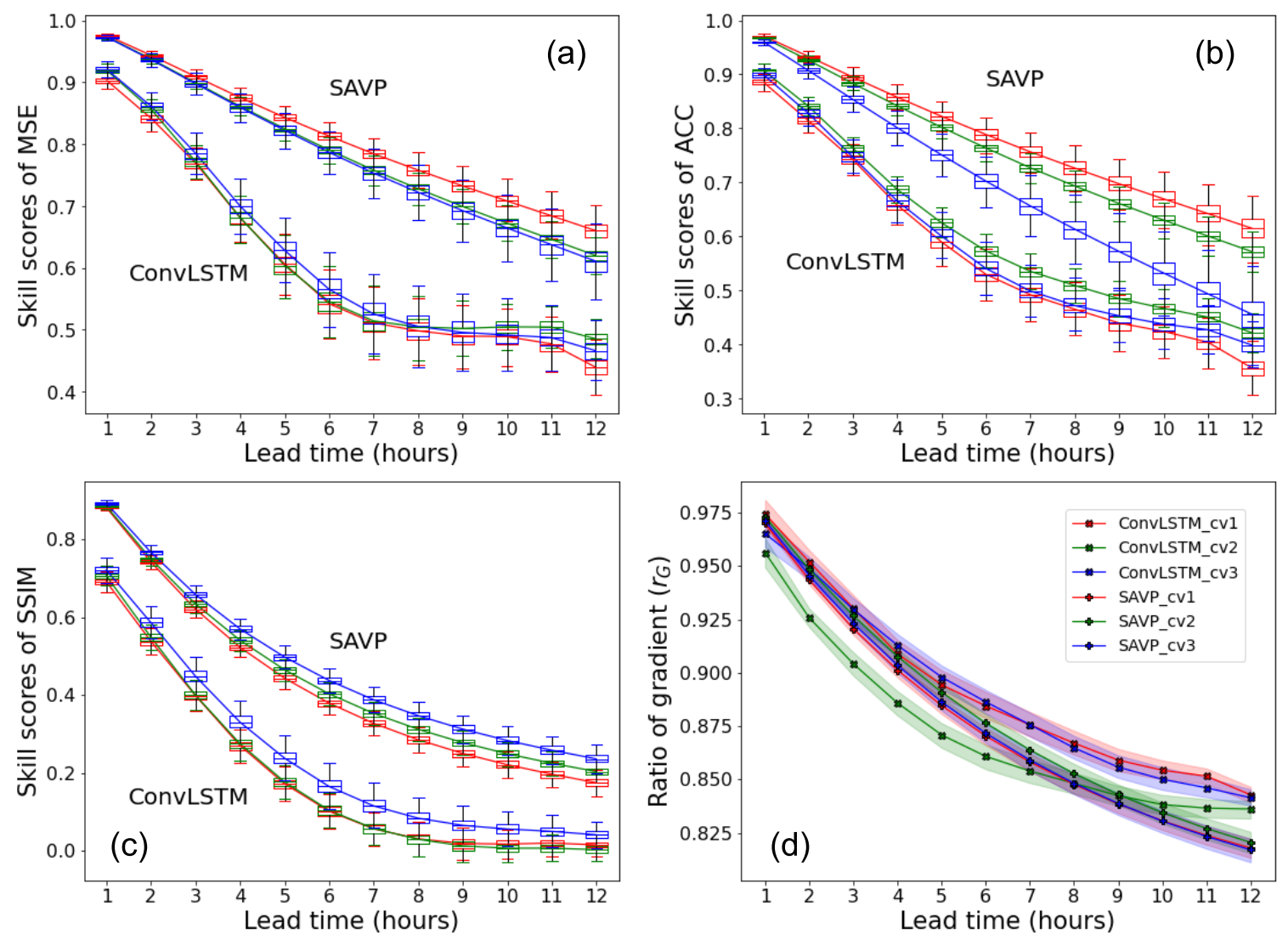

Figure 5. Averaged skill scores of (a) MSE, (b) ACC and (c) SSIM across lead time (x-axis) for the SAVP and the ConvLSTM model. Both models are trained with the three cross-validation datasets (with D1) displayed in Fig. 2. The persistence serves as the reference forecast. Sampling uncertainty estimated via block bootstrapping is illustrated by box-whiskers showing the inter-quartile and -decile range of the skill score values. (d) shows the ratio of the spatially averaged $2 \mathrm{~m}$ temperature gradient for the same video prediction models. The persistence forecasts (not shown) attain a constant value of $r_{G}=1$ since local spatial variability is retained.

reference data for continuous variables by factorizing it into a conditional and a marginal distribution (Murphy and Winkler, 1987; Wilks, 2011). Figure 7 shows the full joint distribution in terms of the calibration-refinement factorization for lead times of 6 and 12 hours. The median as well as the interdecile range of the ground truth conditioned on the forecasts are displayed by the solid and dashed line, respectively. The histogram at log-scale illustrates the marginal distribution of the respective model forecast.

The central parts of the temperature range are well calibrated in both models, but the ConvLSTM-model shows a broader interdecile range in accordance with the larger MSE. Larger deviations from the 1:1-line are obtained near the tails of the conditional distributions in all four panels. Thus, both models have problems to issue calibrated forecasts when the $2 \mathrm{~m}$ temperature is very high (around $310 \mathrm{~K}$ ) or very low (around $250 \mathrm{~K}$ ). It is noteworthy that the marginal distribution of the ConvLSTM model 

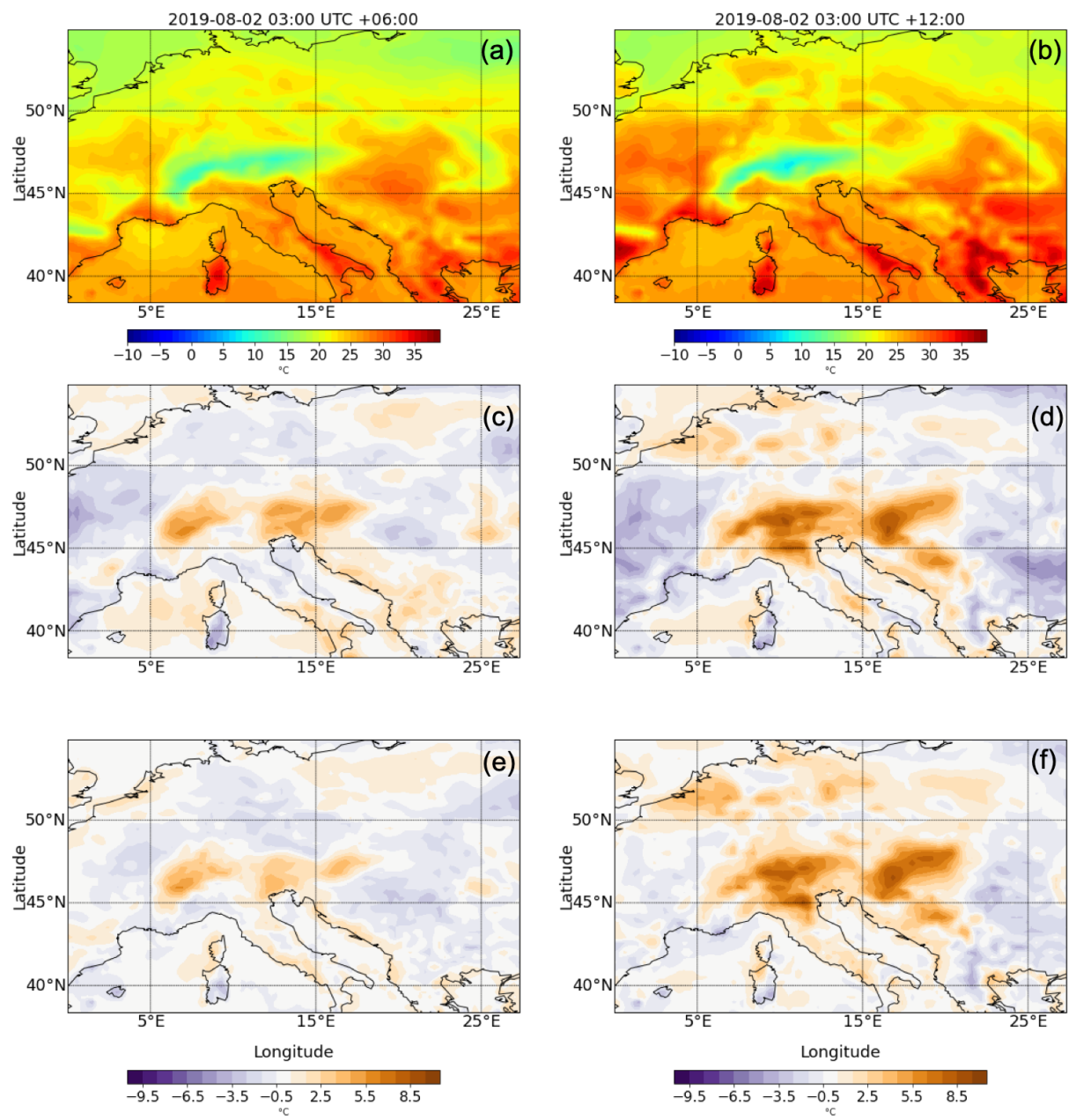

Figure 6. Example forecast for the $2 \mathrm{~m}$ temperature with a lead time of six (first column) and 12 hours (second column). (a,b) The ground truth from the ERA5 reanalysis dataset. (c-f) The difference between the forecasts generated by the ConvLSTM (c,d) and SAVP (e,f) where positive (negative) values represent a warm (cold) bias in the forecasts. The initial time for both model forecasts is 2019-08-02, 03:00 UTC. Further time steps of the 12-hour predictions are provided in Appendix A5.

results becomes narrower for longer lead times since no temperatures below $252 \mathrm{~K}$ (10 out of 8471 samples) are predicted. By contrast, the SAVP-model predicts up to $4 \mathrm{~K}$ colder temperatures even for forecast hour 12, although the forecasts are not well calibrated at the lower tail of the conditional distribution. A similar result, but with a smaller amplitude, can also be deduced at the upper tail of the conditional distributions. Thus, the SAVP-forecasts exhibit a slightly higher degree of refinement, also termed sharpness in statistics (Wilks, 2011), compared to ConvLSTM. 

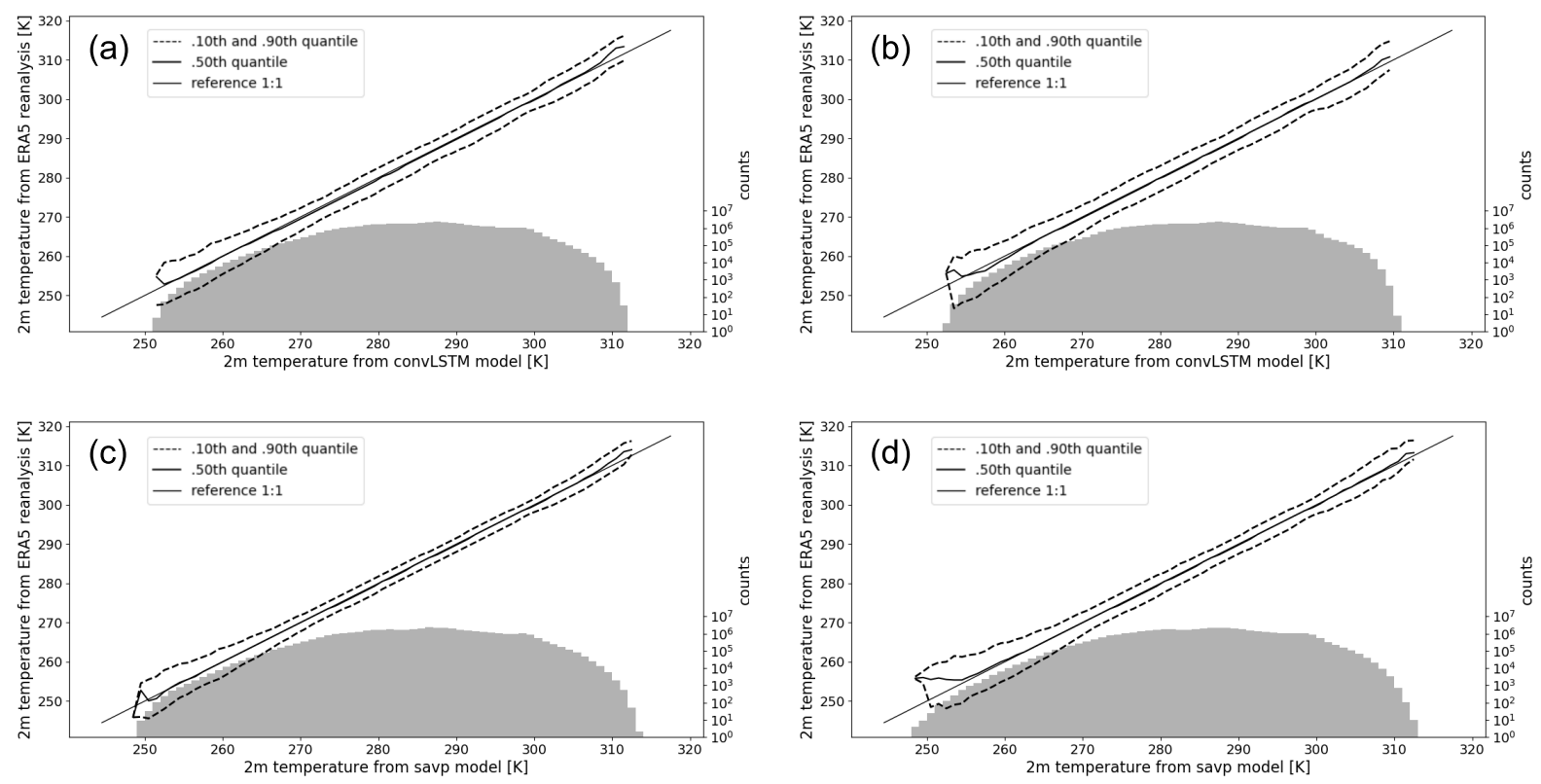

Figure 7. Conditional quantile plots in terms of the calibration-refinement factorization for $2 \mathrm{~m}$ temperature forecasts with a lead time of 6 hours (first column) and 12 hours (second column). (a,b) show the factorization of ConvLSTM forecasts, while (c,d) correspond to the SAVP model. The solid straight line denotes the 1:1 reference line of a hypothetical perfect model. The dash lines represent the $10^{t h}$ and $90^{t h}$ quantiles and the bold solid line represent the median of the ground truth data conditioned on the forecasts, respectively. The marginal distribution of the model forecasts is presented as log histogram (right axis, light grey bars).

\subsection{Trade-off between sharpness and accuracy}

The term "sharpness" has different meanings in the computer vision and meteorological domains. Sharpness in meteorology characterizes the unconditional distribution of the forecasts. A sharp forecast means that the forecasts are frequently enough distinctly different from the climatological value of the predictand. By contrast, sharpness describes the image contrast at the object edges in the computer vision domain. In the following, we discuss sharpness in this latter sense and analyze the local spatial variability of the $2 \mathrm{~m}$ temperature fields in terms of the gradient ratio.

Sensitivity tests were performed by varying the L1-loss scaling factor $\lambda_{1}$ in Eq. (1). While the results are rather insensitive for $\lambda_{1}>100$ (not shown), we notice a stronger dependency of the model performance for smaller values of $\lambda_{1}$. The image sharpness is improved particularly for longer predictions for smaller scaling factors of the L1-loss, while the MSE is slightly increased (see Fig. 8). This implies that the GAN component in SAVP is largely responsible for maintaining the feature contours. By reducing the strong weight of reconstruction loss, the SAVP model is encouraged to produce temperature fields with a higher local variability, although the errors at grid-point level become larger then. Note that such a trade-off between sharpness in terms of the gradient ratio and accuracy in terms of MSE is observed in weather forecast applications as in other computer vision applications (Lee et al., 2018). 

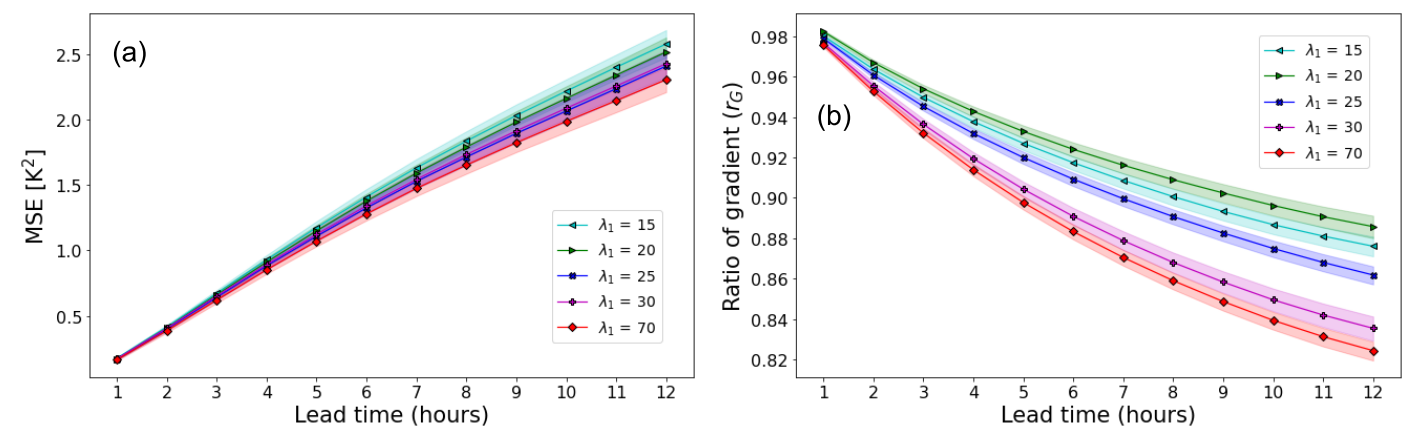

Figure 8. Performance of the SAVP model in terms of (a) MSE and (b) the gradient ratio $r_{G}$ with variations of the scaling factor for the L1-loss $\lambda_{1}$. Note that the results for $\lambda_{1}=70$ only differ marginally from using $\lambda_{1}=10000$.

This sensitivity study also explains why the SAVP in its original configuration does not outperform the ConvLSTM-model in terms of the gradient ratio (Fig. 5). Due to the very large value of the L1 scaling factor $\left(\lambda_{1}=10000\right)$, the model is encouraged to optimize for the pixel-wise loss over other losses (e.g. the adversarial loss). Thus, the out-performance in terms of MSE, ACC and SSIM can be attributed to the more sophisticated generator architecture in SAVP alone rather than the adversarial optimization.

In order to gain additional insight into the performance of the GAN component in SAVP on the tails of the $2 \mathrm{~m}$ temperature distribution, two conditional quantile plots generated with $\lambda_{1}=10000$ and $\lambda_{1}=15$, respectively, are provided in Fig. 9. While there are no significant differences for large parts of the conditional distribution, we observe that the median gets closer to the 1:1 reference line at the lower tail of the PDF for $\lambda_{1}=15$. Thus, lowering $\lambda_{1}$ also yields better calibrated model forecasts for very cold temperatures. Note that the lowest temperatures occur in the Alpine region for our target region where grid points are located more than $2000 \mathrm{~m}$ above sea level (see Figure 1). Since the surface elevation varies quite strongly over the mountainous region, the preservation of large local temperature gradients due to the underlying topography is a necessary prerequisite for well-calibrated forecasts in this region.
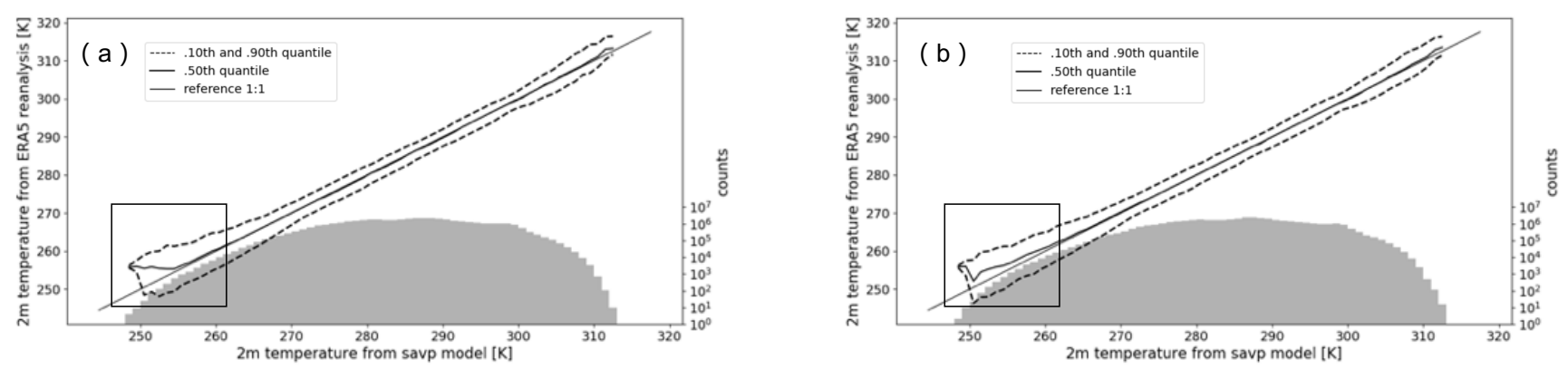

Figure 9. Conditional quantile plots in terms of the calibration-refinement factorization for $2 \mathrm{~m}$ temperature forecasts with a lead time of 12 hours of the SAVP model with (a) $\lambda_{1}=10000$ and (b) $\lambda_{1}=15$, respectively. 
https://doi.org/10.5194/gmd-2021-430

Preprint. Discussion started: 8 March 2022

(c) Author(s) 2022. CC BY 4.0 License.

(c) (i)

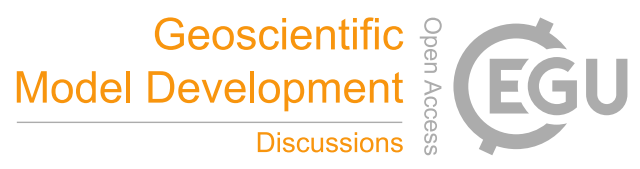

\subsection{Sensitivity analysis}

In the following, we describe further sensitivity tests on the domain size, the selected predictor variables, and the amount of training data of the SAVP model (Experiments 2, 3, and 4, c.f. Tab. A2 in the Appendix). The analysis thereby focuses on evaluating the model performance in terms of the MSE. However, the results of the other evaluation metrics are also briefly presented and the corresponding plots are attached to the Appendix C.

The models in Experiment 2 are trained on data from all 11 years, but the domain size is reduced from $92 \times 56$ to $80 \times 48$ and $72 \times 44$. It is seen that the performance in terms of the MSE slightly deteriorates when the target domain becomes smaller (Fig. 10a). Interestingly, the reduction in MSE is most pronounced when enlarging the target domain from $80 \times 48$ to $92 \times 56$ (reduction in MSE by about $0.2 \mathrm{~K}^{2}$ ). Similar results are obtained in terms of the ACC. However, in terms of the SSIM and the gradient ratio $r_{G}$ only minor changes due to variations in the domain size are observed.

In Experiment 3, the set of predictor variables is reduced gradually from three $\left(T_{2 \mathrm{~m}}, T_{850 \mathrm{hPa}}, T C C\right)$ to two $\left(T_{2 \mathrm{~m}}, T_{850 \mathrm{hPa}}\right)$ and further to one $\left(T_{2 \mathrm{~m}}\right)$. Note that this experiment is conducted on the smaller target domain with $80 \times 48$ grid points on which the average MSE tracks about $0.2 \mathrm{~K}^{2}$ higher than on the largest domain with $92 \times 56$ grid points. While the MSE is fairly insensitive with respect to the inclusion of the TCC, a significant increase is observed when $T_{850 \mathrm{hPa}}$ is dropped from the list of predictors. For the former case, the MSE is just increased by about $0.1 \mathrm{~K}^{2}$, whereas the latter results in a MSE of $3.7 \mathrm{~K}^{2}$ after a lead time of 12 hours. Thus, the temperature of the airmass is more relevant than the total cloud cover for predicting the diurnal cycle of $2 \mathrm{~m}$ temperature in our study. In terms of the other evaluation metrics (ACC, SSIM and $r_{G}$ ), similar results are obtained.

To evaluate the impact of the amount of training samples on the forecasting performance, the size of the training dataset is reduced from 11 years in D1 to 10 years in D2 and eight years in D3, respectively (see Fig. 2). Removing a single year from the training dataset does not affect the model performance in terms of the MSE (Fig. 10c). When three years of data are dropped from the training dataset, we notice a slight deterioration (MSE increases to about $2.45 \mathrm{~K}^{2}$ from $2.3 \mathrm{~K}^{2}$ ). In terms of the ACC, the SSIM and $r_{G}$, the model performance is also rather insensitive against the variations in the size of the dataset probed in this 415 study.

\section{Discussion}

The results presented in the previous section demonstrate that video frame prediction models from computer vision attain some predictive skill in forecasting the diurnal cycle of $2 \mathrm{~m}$ temperature. We showed that the SAVP model performs significantly better than a simple ConvLSTM model in terms of several evaluation metrics (MSE, ACC and SSIM). This confirms our expectation that more advanced DL can better extract spatio-temporal features from the input sequence to predict the future state, which in turn is beneficial for meteorological applications, even though these models are originally developed for applications in computer vision.

However, the local spatial variability as measured in terms of the gradient ratio is not necessarily improved in our experiments with the SAVP model. Experiments with varied scaling factors of the L1-loss component $\lambda_{1}$ reveal that the strong weight on 

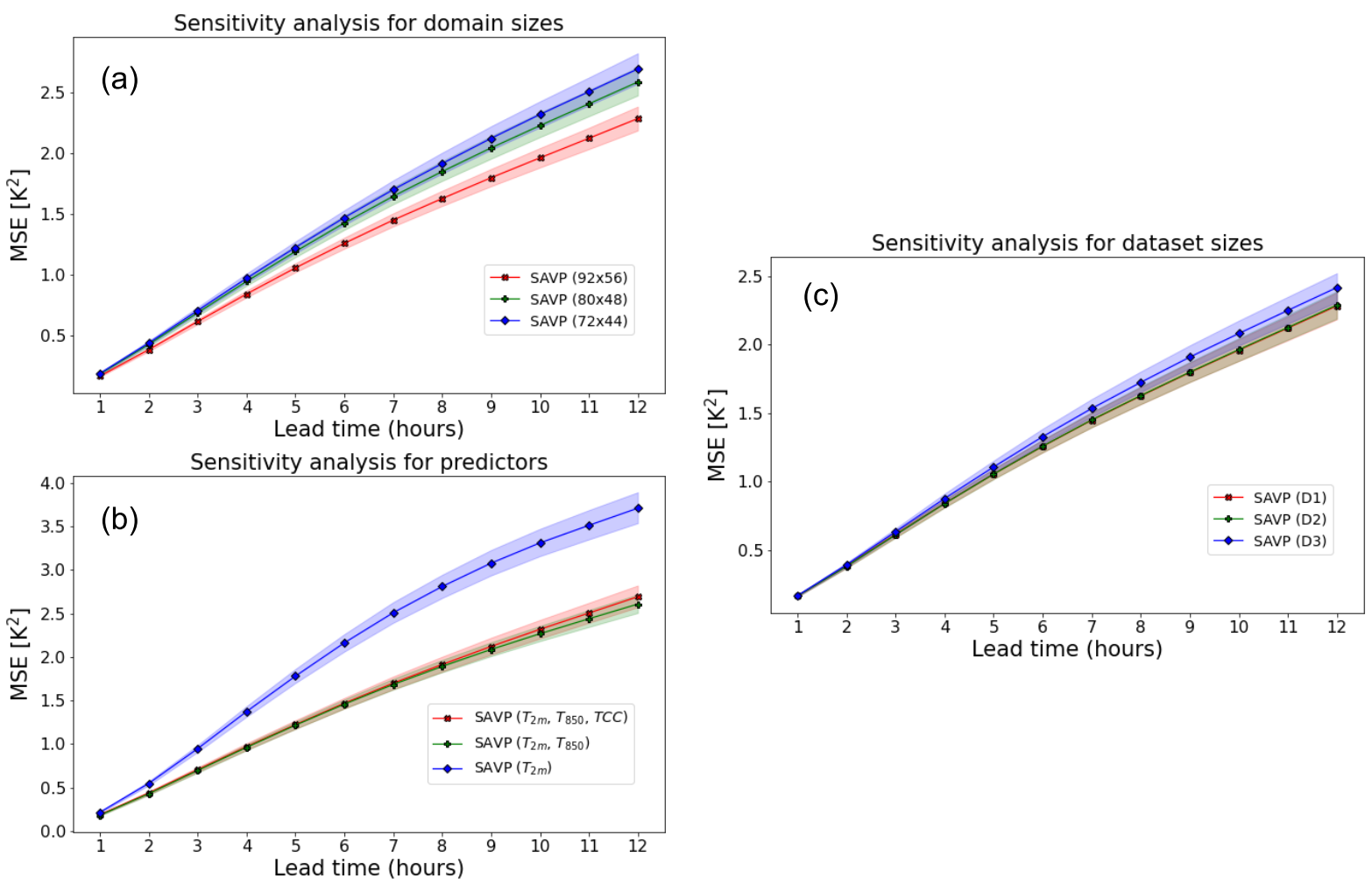

Figure 10. Averaged MSE of $2 \mathrm{~m}$ temperature forecasts with the SAVP model for different sensitive experiments using (a) different domain sizes (red $-92 \times 56$, green $-80 \times 48$, blue $-72 \times 44$ ), (b) different predictors (red $-T_{2 \mathrm{~m}}, T_{850 \mathrm{hPa}}$ and $T C C$, green $-T_{2 \mathrm{~m}}, T_{850 \mathrm{hPa}}$, blue $T_{2 \mathrm{~m}}$ ) and (c) different sizes of the training dataset (red - 11 years, green - 10 years, blue -8 years). The sampling uncertainty estimated via block bootstrapping is denoted by colour shading.

the pixel-wise reconstruction loss in our basic hyperparameter setting (cf. Section 3.3.2) is responsible for this behaviour. With $\lambda_{1}=10000$, the adversarial part of the SAVP architecture is effectively neglected. Thus, the improvement seen in the evaluation metrics can be attributed to a more advanced generator which incorporates ConvLSTM cells, several convolutional layers along with skip connections and conditioning information on latent code. Reducing $\lambda_{1}$, the accuracy of the model in terms of the MSE slightly decreases, but the local spatial variability becomes much more similar to the ground truth data. In other words, the adversarial training with the GAN-components encourages the model to preserve local features in the $2 \mathrm{~m}$ temperature field which can be attributed to spatial variability due to varying characteristics of the Earth's surface. Note that the latter characteristics (e.g. land-sea contrast and surface elevation) has not been explicitly fed to the models, so that their impact needed to be learnt in a data-driven way. Additionally, the forecasts tend to be better calibrated for very cold conditions. Since very cold conditions constitute the tail of the marginal distribution of $2 \mathrm{~m}$ temperature, it is hypothesized that the GAN-component in SAVP may help to forecast extreme temperature events. 
https://doi.org/10.5194/gmd-2021-430

Preprint. Discussion started: 8 March 2022

(c) Author(s) 2022. CC BY 4.0 License.

(c) (i)

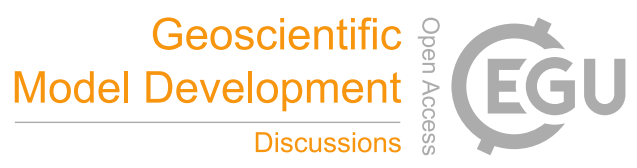

Further sensitivity experiments reveal that the prediction of the diurnal cycle of $2 \mathrm{~m}$ temperature can significantly benefit from incorporating additional predictors. In particular, the temperature at $850 \mathrm{hPa}$ provides additional information on the air mass characteristics which in turn yield a strong reduction of the MSE by at least $30 \%$. However, adding the total cloud cover surprisingly barely contributed although cloud drive the energy fluxes at the surface which in turn drive the diurnal cycle of near surface temperature and the planetary boundary layer in general (e.g. von Engeln and Teixeira, 2013; Chepfer et al., 2019). One reason for this result might be that the model has problems in predicting the future cloud cover since the underlying microphysical processes are highly complex (see, e.g., Khain et al., 2015) and that we only optimize the model on the $2 \mathrm{~m}$ temperature. While this turned out to be beneficial in our study (not shown), meaningful feature abstraction from quickly varying predictors becomes challenging.

Furthermore, the model performance is slightly improved upon enlarging the target domain. On the one hand side, this might be attributed to an improved feature abstraction of the large-scale atmospheric conditions (e.g. the advection of air masses). On the other hand, the synoptic-scale features have limited relevance at sub-daily time scales since the time and spatial scales of atmospheric processes are correlated (see, e.g. Orlanski, 1975). Besides, the largest domain $92 \times 56$ includes the largest fraction of marine pixels. Since the $2 \mathrm{~m}$ temperature exhibits much smaller diurnal variations over the sea surface (Ginzburg et al., 2007), the prediction becomes simpler for these regions which in turn translate to smaller prediction errors (see also Fig. 6).

In addition, the MSE of the SAVP model trained with 11 years of data is slightly decreased, but compared to the additional amount of data included (37.5\% compared to the 8 years-dataset), the effect is judged to be minor. We argue that the dataset should probably not include less data than probed here, but contrarily, including more data from the ERA5 reanalysis database is not expected to provide substantial benefits. It could even be that stronger climate change signals may outweigh the added value of including more data to the test dataset.

Our study shares some similarities with the study of Bihlo (2020) which also presents short-range forecasts of the $2 \mathrm{~m}$ temperature over Europe with a GAN-based model. While his predictions attain a fairly low RMSE of about $0.53 \mathrm{~K}$ for 12-hour forecasts which is considerably smaller than the model performance with our SAVP model (RMSE $(S A V P)=$ $\sqrt{M S E(S A V P)} \simeq 1.5 \mathrm{~K})$, direct comparison is limited due to relevant changes in the target of the forecast product. First, the spatial resolution of the target product is higher with $0.3^{\circ}$ compared to $0.5^{\circ}$ and thus, local spatial variability must be captured more precisely in our case study. Second, we choose a hourly forecast product to focus explicitly on the predictability on the diurnal cycle. Thus, twelve consecutive forecasts are required to generate a 12-hour forecast with our SAVP which is considerably more than four time steps in Bihlo (2020). Thus, the increased temporal resolution of our forecasts come at the price of a stronger error accumulation, since the forecasts are conditioned on the previous hour.

Furthermore, we notice that the gap between data-driven neural networks for meteorological forecasts and contemporary NWP models is still considerable. While a RMSE of $1.5 \mathrm{~K}$ for a 12-hour forecasts, is attained with our SAVP model, contemporary global NWP models show up with a RMSE of about $0.4 \mathrm{~K}^{1}$. Meanwhile, they also provide a higher spatial solutions around $0.1^{\circ} \simeq 10 \mathrm{~km}$. However, in light of the long development history of NWP models for several decades (Bauer et al.,

\footnotetext{
${ }^{1}$ This value was extracted on 2021-12-10 from the NCEP/EMC global model verification website.
} 
https://doi.org/10.5194/gmd-2021-430

Preprint. Discussion started: 8 March 2022

(c) Author(s) 2022. CC BY 4.0 License.

(c) (i)

2015), the results with data-driven neural networks is already encouraging. Thus, further research as presented in the following section, may further close the gap between deep neural networks and classical NWP models.

\section{Conclusion}

In this study, we have explored the application of video prediction models, originally developed for computer vision applications, to forecast the sub-daily temperature evolution over Europe. While the results show that more sophisticated model architectures such as the SAVP model and the inclusion of informative predictors such as the $850 \mathrm{hPa}$ temperature can improve significantly the model performance, we also shed further light on the orchestration of different loss components in the composite SAVP architecture. Tuning the model on the L1-loss optimizes for the MSE, but also leads to a strongly underestimated local spatial variability. Conversely, choosing a smaller weight on the L1-loss leads to slight increase in MSE, but the spatial variability gets better preserved due to a relatively stronger contribution of the GAN-component in SAVP.

The findings in our study and the persisting large gap to NWP models motivate motivate future work which aiming to improve the performance of the underlying deep neural networks. First, one may consider testing further state-of-the-art video prediction models from computer vision which continue to develop at quick momentum. Further advanced GAN-based models (e.g Brock et al., 2019; Clark et al., 2019; Qi et al., 2020) or the recent success in vision transformers (e.g. Caron et al., 2021; Yan et al., 2021) are appealing candidates which may help to reduce the above mentioned gap. Apart from improving the model architecture, our results also suggest that deep neural can benefit greatly from adding further predictors beside the target variable to further improve the forecast skill. In our case, the $850 \mathrm{hPa}$ temperature proved to be beneficial for the model performance and it is likely that other dynamic predictors such as surface fluxes or near-surface wind can contribute to the model performance as well. Also static fields such as surface elevation and the land-sea mask should be considered (Sha et al., 2020; Lezama Valdes et al., 2021). Thus, a more systematic predictor selection is an appealing candidate to further improve the forecast skill.

Another way would be to exploit explicitly physical knowledge. This could be realized during preprocessing via feature engineering or during training by formulating physical constraints (de Bézenac et al., 2019; Karniadakis et al., 2021). In some cases, even simple physical constraints can be beneficial and can furthermore increase the realism when predictions beyond the training data space need to be issued (Karpatne et al., 2017).

495 Additionally, enlarging the forecast domain is considered to be helpful, especially when the lead time is extended. For medium-range forecasts, even a processing of the global atmospheric state, but it is mentioned that this would result into enormous memory requirements on the operating GPU used for training, at least when a highly resolved forecast product is demanded (c.f. Dueben and Bauer, 2018).

The SAVP model can also be used for probabilistic forecasting due to the involved GAN and VAE components. Due to the multi-scale, non-linear interactions in atmospheric processes, of which the natural uncertainty is large (see, e.g., Lorenz, 1969) and quantifying this uncertainty is considered to be crucial in meteorology (Vannitsem et al., 2021). The demand for a probabilistic framework further increases when the forecast product involves higher inherent uncertainty than the $2 \mathrm{~m}$ temperature. 
https://doi.org/10.5194/gmd-2021-430

Preprint. Discussion started: 8 March 2022

(c) Author(s) 2022. CC BY 4.0 License.

(c) (i)

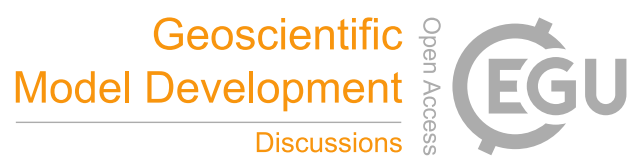

Precipitation is a typical example for such a meteorological quantity since it is subject to complex interacting microphysical and dynamical processes on small spatio-temporal scales (Sun et al., 2014). While precipitation nowcasting is already gaining momentum in the meteorological community (Prudden et al., 2020), video prediction models may also be helpful to extent the forecast range beyond a few hours. This is motivated by the fact that even contemporary NWP models still have problems in predicting precipitation events, while first DL-based applications already start to compete successfully with these models (see, e.g., Espeholt et al., 2021).

Code and data availability. The current version of model discribed in the paper is available from the project website: https://gitlab.jsc.fz-

510 juelich.de/esde/machine-learning/ambs///tree/GMD1 under the MIT licence. The exact version of the model used to produce the results used in this paper is archived on zenodo (https://doi.org/10.5281/zenodo.6308774). The scripts can run the model and make the plots for all the simulations presented in this paper.

\section{Appendix A: Experimental setup}

As shown in Table A2, we conducted four experiments with different settings, i.e. varying input variables, regions, and number of training samples, to explore the feasibility and robustness capability by DL for $2 \mathrm{~m}$ temperature forecasting. We finally obtain eight datasets as list in Table A1, which will be adapted for different experiments in Table A2.

\section{Appendix B: Evaluation metrics}

In this section, we provide some mathematical details on the evaluation metrics used in this study, that are the mean squared error (MSE), the anamoly correlation coefficient (ACC), the Structural Similarity Index (SSMI) and the gradient ration $r_{G}$.

\section{B1 Mean squared error (MSE)}

The MSE measures the squared difference between the model data and the ground truth data. Let $x_{i j}$ and $\hat{x}_{i j}$ constitute data on discrete grid points of the ground truth and the forecasts, respectively, where the grid consists of $I$ and $J$ cellcenter positions in zonal and meridional direction (or the width and height in pixels for images), respectively. With $N=I+J$, the MSE reads

$$
M S E=\frac{1}{N} \sum_{i=1}^{I} \sum_{j=1}^{J}\left[\hat{x}_{i j}-x_{i j}\right]^{2} .
$$


Table A1. Overview on the datasets used in this study. Note: D1, D2, D3 correspond to the three datasets where the number of years in the training dataset is varied $(11,10$, and eight years, respectively) as illustrated in Fig. 2.

\begin{tabular}{llll}
\hline \hline Dataset ID & Training Dataset & Domain size & Variables \\
\hline \hline 1 & D1 & $92 \times 56$ & TCC, $T_{850 \mathrm{hPa}}, T_{2 \mathrm{~m}}$ \\
\hline 2 & D2 & $92 \times 56$ & TCC, $T_{850 \mathrm{hPa}}, T_{2 \mathrm{~m}}$ \\
\hline 3 & D3 & $92 \times 56$ & TCC, $T_{850 \mathrm{hPa}}, T_{2 \mathrm{~m}}$ \\
\hline 4 & D2 & $80 \times 48$ & TCC, $T_{850 \mathrm{hPa}}, T_{2 \mathrm{~m}}$ \\
\hline 5 & D2 & $72 \times 44$ & TCC, $T_{850 \mathrm{hPa}}, T_{2 \mathrm{~m}}$ \\
\hline 6 & D2 & $80 \times 48$ & $T_{850 \mathrm{hPa}}, T_{2 \mathrm{~m}}$ \\
\hline 7 & D2 & $80 \times 48$ & $T_{2 \mathrm{~m}}$ \\
\hline
\end{tabular}

Table A2. Overview on the experiments in this study.

\begin{tabular}{llll}
\hline \hline Experiments IDs & Dataset IDs & Models & Target (Scientific questions to be answered) \\
\hline \hline 1 & 1 & ConvLSTM, SAVP, Persistence & Test and compare models performances \\
\hline 2 & $1,4,5$ & SAVP & Test sensitivity to the domains \\
\hline 3 & $4,6,7$ & SAVP & Test sensitivity to the variables \\
\hline 4 & $1,2,3$ & SAVP & Test sensitivity to the size of training data \\
\hline 5 & 1 & SAVP & Sensitivity to the scaling factor $\lambda_{1}$ \\
\hline
\end{tabular}

\section{B2 Anomaly correlation coefficient (ACC)}

The ACC quantifies how well the spatial position of anomalies matches between the predicted and the ground truth data (i.e. the ERA5 reanalysis data in our case). The uncentered ACC is given by:

$$
A C C=\frac{\sum_{i=1}^{I} \sum_{j=1}^{J}\left[\hat{x}_{i j}-x_{i j}^{c}\right]\left[x_{i j}-x_{i j}^{c}\right]}{\left\{\sum_{i=1}^{I} \sum_{j=1}^{J}\left[\hat{x}_{i j}-x_{i j}^{c}\right]^{2} \sum_{i=1}^{I} \sum_{j=1}^{J}\left[x_{i j}-x_{i j}^{c}\right]^{2}\right\}^{1 / 2}} .
$$

Here, $x_{i j}^{c}$ represents the climatological mean which is inferred at each grid point from the ERA5 reanalysis data between 1990 and 2019 in this study. Since the $2 \mathrm{~m}$ temperature involves a seasonal and a diurnal cycle, the climatology is calculated separately for each month of the year and each of the day, respectively. 
https://doi.org/10.5194/gmd-2021-430

Preprint. Discussion started: 8 March 2022

(c) Author(s) 2022. CC BY 4.0 License.

(c) (i)

\section{B3 Structural Similarity Index (SSIM)}

The SSIM constitutes a score metric typically applied in computer vision to measure the similarity between two images (Wang et al., 2004). It measures and compares the structural information between the ground truth and prediction images. The similarity is thereby quantified in terms of luminance, contrast variance and structure. In case of images with multiple channels (i.e. RGB-images), the calculations are done separately for each channel and averaged afterwards.

- Luminance: The luminance is measured by averaging the pixels' brightness of the images. Letting $\mu_{\mathbf{X}}$ and $\mu_{\hat{X}}$ denote the averaged brightness of the pixels from the ground truth and the generated image, the respective component $l$ of SSIM is given by

$$
l=\frac{2 \mu_{X} \mu_{\hat{X}}+C_{1}}{\mu_{X}^{2}+\mu_{\hat{X}}^{2}+C_{1}}
$$

where $C_{1}$ is a constant to avoid divisions by zero or very small numbers. Specifically, we choose $C_{1}=\left(K_{1} L\right)^{2}$ where $K_{1}=0.01$ and $L$ is the dynamic range of input values. In case that the average brightness of the two images matches, $l=1$ is obtained.

- Contrast: The contrast is measured by calculating separately the standard deviation of the pixel brightness of each image. Letting $\sigma_{X}$ and $\sigma_{\hat{X}}$ denote the standard deviation of the ground truth and the generated image with

$$
\sigma_{X}=\left[\frac{1}{N-1} \sum_{i=1}^{I} \sum_{j=1}^{J}\left(x_{i j}-\mu_{X}\right)^{2}\right]^{1 / 2} .
$$

The contrast score component $c$ then reads:

$$
c=\frac{2 \sigma_{X} \sigma_{\hat{X}}+C_{2}}{\sigma_{X}^{2}+\sigma_{\hat{X}}^{2}+C_{2}}
$$

$C_{2}$ is added in analogy to $C_{1}$, but for the contrast score component. Here, $C_{2}=\left(K_{2} L\right)^{2}$ and $K_{1}=0.03$.

- Structure: The structure is computed with the help of the covariance in pixel-space between the ground truth and the generated image and therefore measures the coherence between the two images in terms of variations around their average brightness. With $\sigma_{X \hat{X}}$ denoting the covariance and using the standard deviations of the ground truth and generated image, that is $\sigma_{X}$ and $\sigma_{\hat{X}}$, the structural component $s$ of SSIM is defined by

$$
s=\frac{\sigma_{X \hat{X}}+C_{3}}{\sigma_{X} \sigma_{\hat{X}}+C_{3}}
$$

where $C_{3}=C_{2} / 2$ serves as an additional constant, as proposed in the study Wang et al. (2004).

Finally, the SSIM is obtained by merging the different components together with the help of

$$
S S I M=l^{\alpha} \cdot c^{\beta} \cdot s^{\gamma} .
$$


Here, $\alpha, \beta$ and $\gamma$ are disposal (positive) parameters which control the importance of each score component. Here, we use $\alpha=\beta=\gamma_{c}=1$. In our particular translation, the luminence corresponds to a measure which compares the domain-averaged $2 \mathrm{~m}$ temperature between prediction and the respective ERA5 reanalysis data. Contrast is equivalent to an comparison between the global variability of $T_{2 \mathrm{~m}}$ per time step, while the structure simply compares the covariance between the predicted and the ground truth $2 \mathrm{~m}$ temperature fields.

\section{B4 Gradient ratio}

The SSIM does not account explicitly account for local variability in the data. Indeed, the $l$ and $c$ components only evaluate the overall average and the variability. Only $s$ measures how the data varies on a grid point (pixel-wise) level. To further analyze the local spatial variability, we calculate the amplitude of the horizontal $2 \mathrm{~m}$ temperature gradient. Sha et al. (2020) follow a similar approach in their temperature downscaling application, but rather deploy the Laplace operator than the gradient operator. Besides, their operator does not account for the curvature on the sphere.

In the geographical coordinate system, the horizontal gradient of the arbitrary quantity $\psi$ reads:

$$
\nabla_{h} \psi=\frac{1}{r_{E} \cos (\varphi)} \frac{\partial \psi}{\partial \lambda} \mathbf{e}_{\lambda}+\frac{1}{r_{E}} \frac{\partial \psi}{\partial \varphi} \mathbf{e}_{\varphi}
$$

Here, $\lambda$ and $\varphi$ denote the longitude and latitude, while $r_{E}$ is the (averaged) Earth radius.

On a geographical grid, the amplitude of the continuous gradient operator $\nabla_{h}$ can be discretized with finite differences:

$$
G_{\psi}=\left|\nabla_{h} \psi(i, j)\right| \simeq \frac{1}{r_{E}} \sqrt{\frac{1}{\cos ^{2}(\varphi(j))}\left(\frac{\psi(i+1, j)-\psi(i-1, j)}{2 \Delta \lambda}\right)^{2}+\left(\frac{\psi(i, j+1)-\psi(i, j-1)}{2 \Delta \varphi}\right)^{2}},
$$

where $\Delta \lambda$ and $\Delta \varphi$ denote the grid spacing of the underlying grid. $i$ and $j$ correspond to indices on the grid in zonal and meridional direction, respectively. This yields a second-order accurate discretization on a regular grid (constant spacing in horizontal directions).

Now, let $G_{X}$ and $G_{\hat{X}}$ denote the average of the absolute horizontal gradient of our target quantity over all grid points on the domain (apart from the lateral boundaries) for the ground truth and the predicted data, respectively. The ratio

$$
r_{G}=\frac{G_{\hat{X}}}{G_{X}}
$$

then measures the averaged local spatial variability amplitude in the predicted field compared to the reference data. For $r_{G}=1$, the amplitude of the horizontal gradient is on average the same in the prediction and in the ground truth. For $r_{G}<1\left(r_{G}>1\right)$, the horizontal gradient is on average underestimated (overestimated) by the model, indicating that the field is visually too blurried (too sharp) following the discussion in Section 3.

\section{Appendix C: Supplementary figures}


https://doi.org/10.5194/gmd-2021-430

Preprint. Discussion started: 8 March 2022

(c) Author(s) 2022. CC BY 4.0 License.

(c) (i)
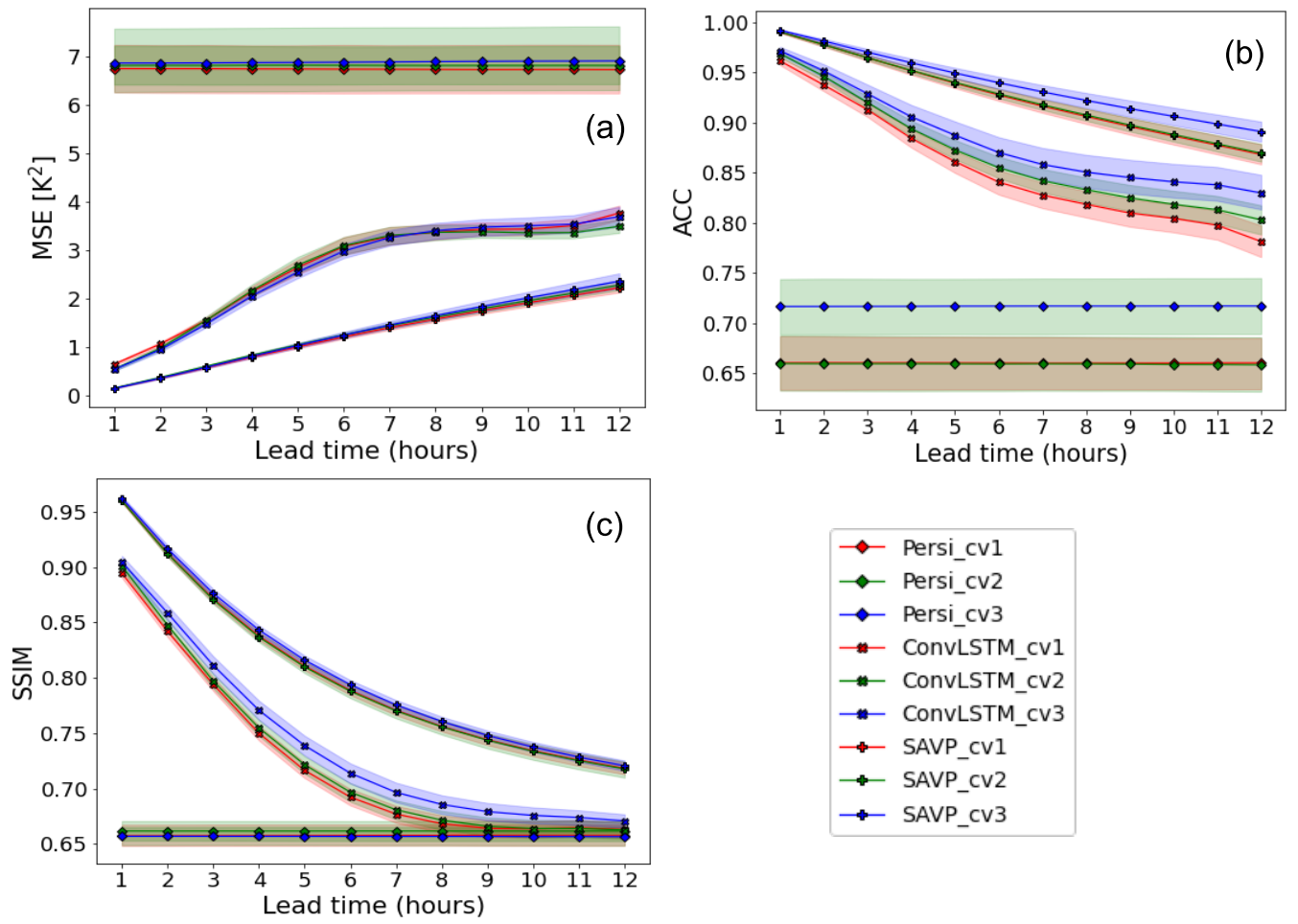

Figure A1. Averaged (a) MSE, (b) ACC and (c) SSIM across lead time ( $x$-axis) for the SAVP, the ConvLSTM and the persistence forecasts from Experiment 1 in Table A2. Like in Fig. 2, sampling uncertainty is estimated via block bootstrapping. However, the interdecile confidence range is now colour shaded for the three cross validation datasets using different models. 
https://doi.org/10.5194/gmd-2021-430

Preprint. Discussion started: 8 March 2022

(c) Author(s) 2022. CC BY 4.0 License.

(c) (i)
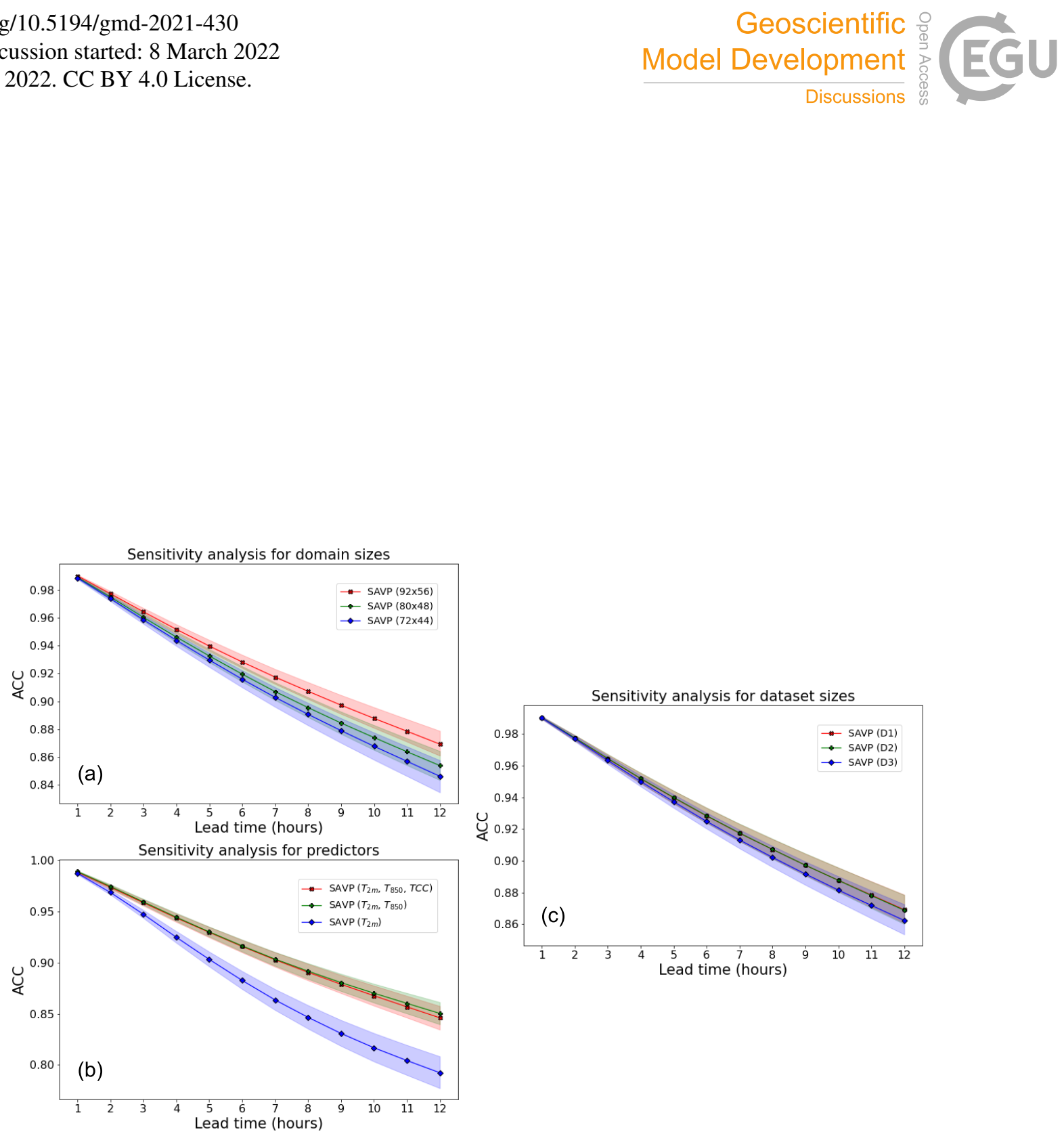

Figure A2. ACC over the forecast period for $2 \mathrm{~m}$ temperature with interdecile bootstrap confidence intervals (shading area) using the SAVP model for different sensitive experiments using (a) different domain sizes (red $-92 \times 56$, green $-80 \times 48$, blue $-72 \times 44$ ), (b) different predictors (red $-T_{2 \mathrm{~m}}, T_{850 \mathrm{hPa}}$ and $T C C$, green $-T_{2 \mathrm{~m}}, T_{850 \mathrm{hPa}}$, blue $-T_{2 \mathrm{~m}}$ ) and (c) different sizes of the training dataset (red -11 years, green -10 years, blue -8 years). 
https://doi.org/10.5194/gmd-2021-430

Preprint. Discussion started: 8 March 2022

(c) Author(s) 2022. CC BY 4.0 License.

(c) (i)
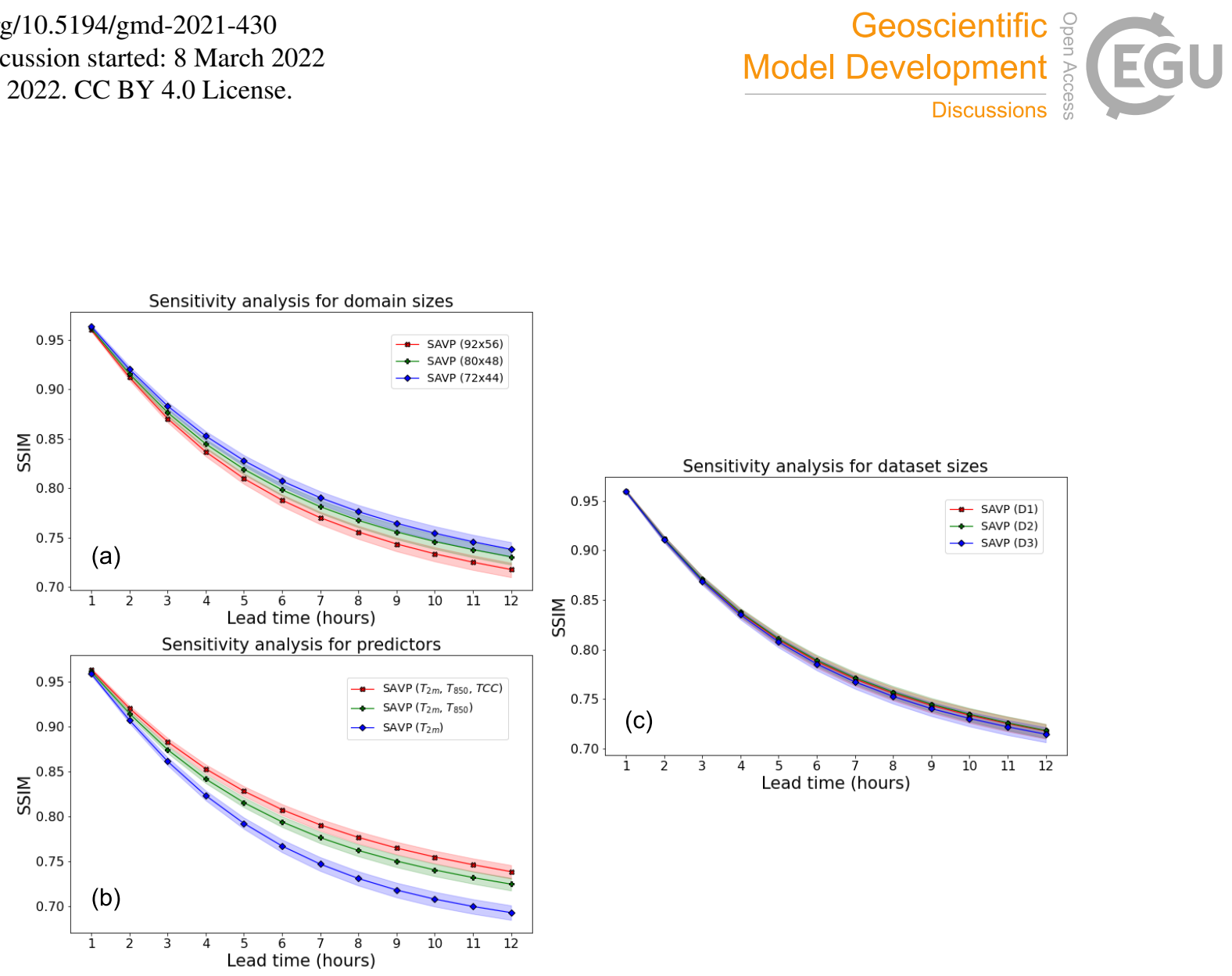

Figure A3. Like Fig. A2, but for SSIM.
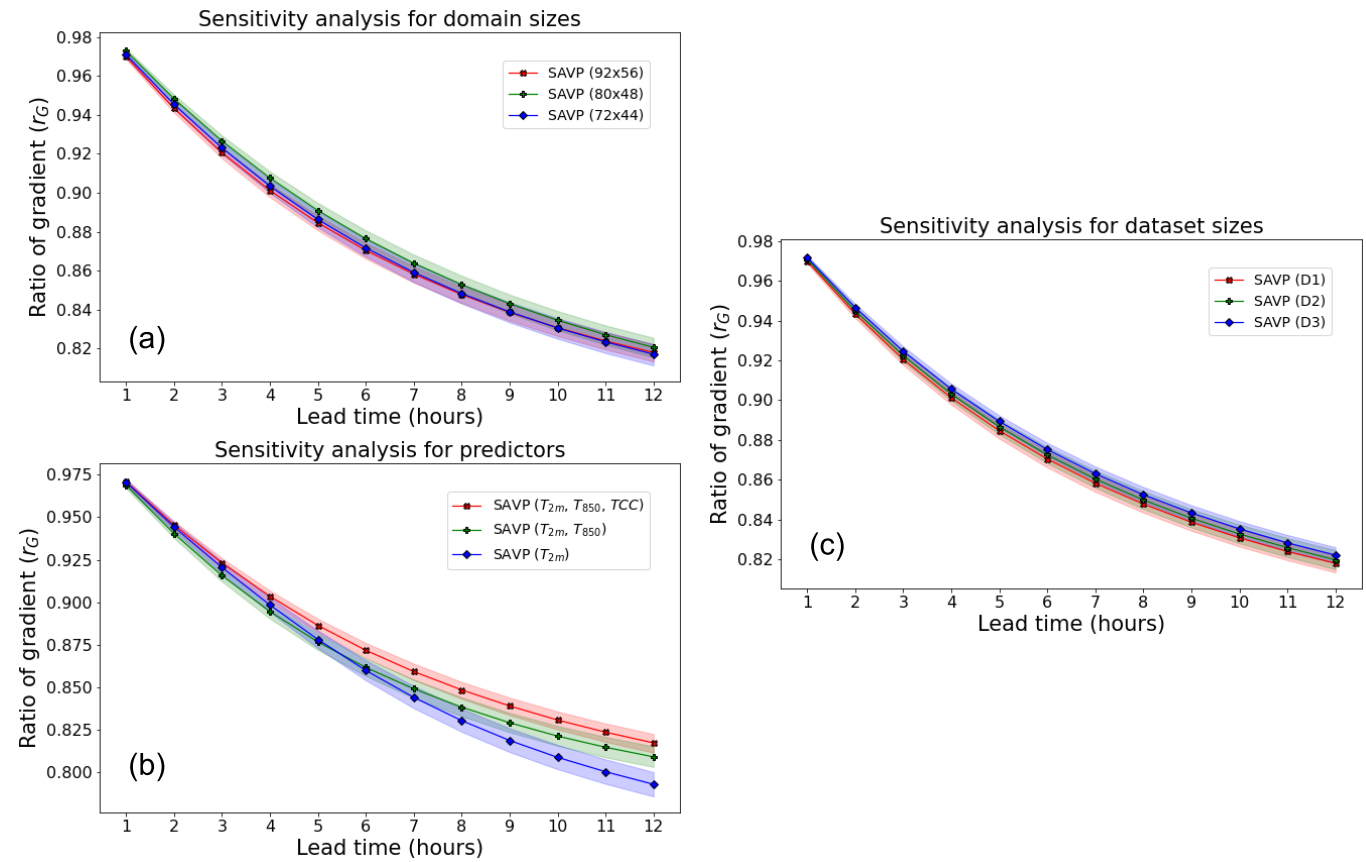

Figure A4. Like Fig. A2, but for the gradient ratio $r_{G}$. 
https://doi.org/10.5194/gmd-2021-430

Preprint. Discussion started: 8 March 2022

(c) Author(s) 2022. CC BY 4.0 License.
Geoscientific Model Development

Discussions
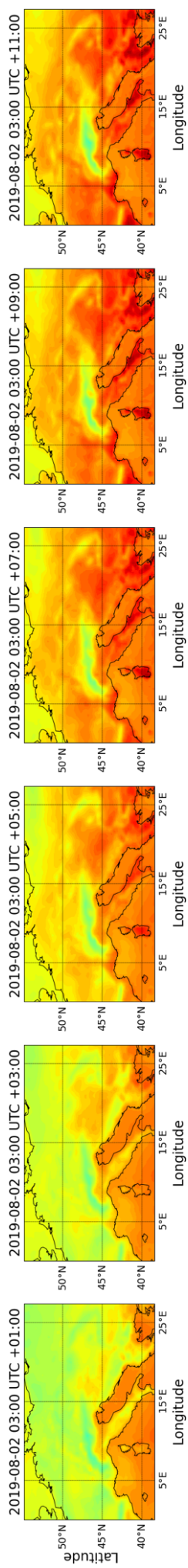
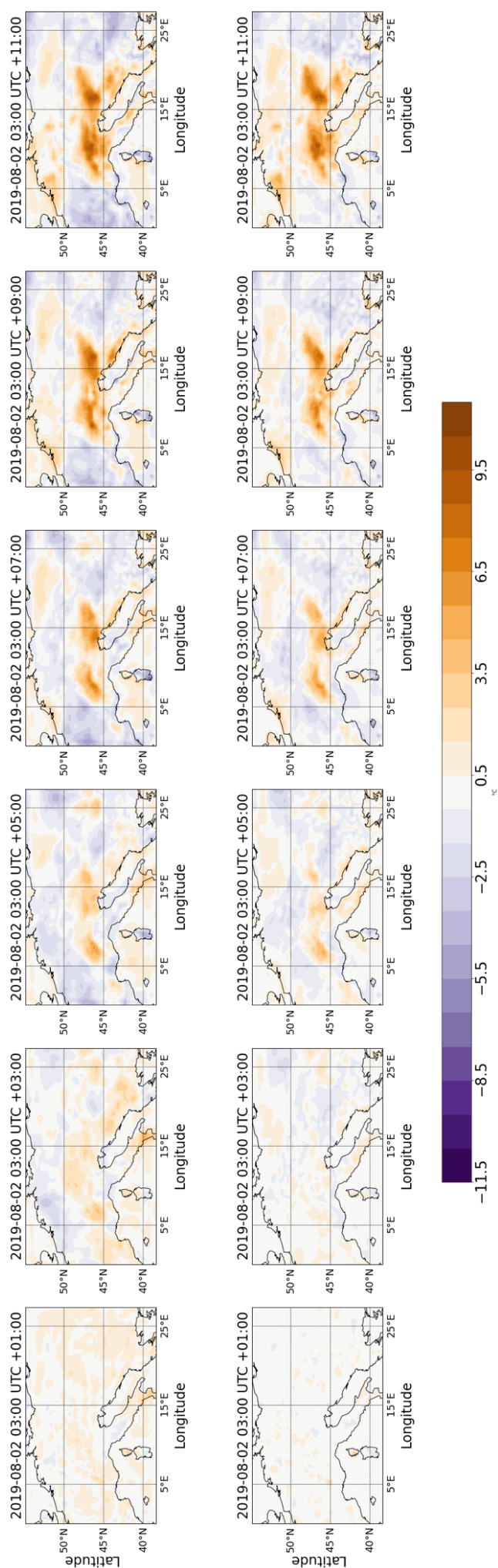

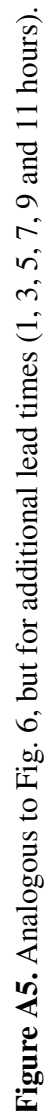


https://doi.org/10.5194/gmd-2021-430

Preprint. Discussion started: 8 March 2022

(c) Author(s) 2022. CC BY 4.0 License.

(c) (1)

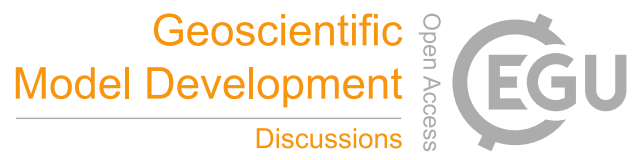

Author contributions. Author contributions. Bing Gong and Michael Langguth equally contributed to this work and performed the bulk of the coding, the experiments, the analysis, and the writing. The study was conceived by Martin Schultz and Bing Gong. Bing Gong, Michael Langguth, Yan Ji, contributed to the method development and maintain the codes. All authors have reviewed and edited the manuscript in several iterations. Martin Schultz supervised the entire project and secured funding.

Competing interests. The authors declare that they have no conflict of interest

Acknowledgements. The authors acknowledge funding from the DeepRain project under grant agreement 01 IS18047A from the Bundesministerium für Bildung und Forschung (BMBF), from the European Union H2020 MAELSTROM project (grant No. 955513, co-funding by BMBF), and from the ERC Advanced grant IntelliAQ (grant no. 787576). We thank Olaf Stein and Lars Hoffmann for preparing the datasets

595 used in our research, as well as Severin Hußmann for an initial attempt to apply video prediction techniques to weather forecasting and helpful scientific discussions. The authors also gratefully acknowledge the Helmholtz Association's Earth System Modelling Project (ESM) for funding this work by providing computing time on the ESM partition of the supercomputer JUWELS as it described by Jülich Supercomputing Centre (2019) at the Jülich Supercomputing Centre (JSC). 
https://doi.org/10.5194/gmd-2021-430

Preprint. Discussion started: 8 March 2022

(c) Author(s) 2022. CC BY 4.0 License.

(c) (i)

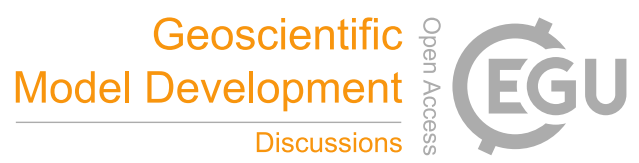

\section{References}

Ayzel, G., Heistermann, M., and Winterrath, T.: Optical flow models as an open benchmark for radar-based precipitation nowcasting (rainymotion v0. 1), Geoscientific Model Development, 12, 1387-1402, 2019.

Ayzel, G., Scheffer, T., and Heistermann, M.: RainNet v1. 0: a convolutional neural network for radar-based precipitation nowcasting, Geoscientific Model Development, 13, 2631-2644, 2020.

Bauer, P., Thorpe, A., and Brunet, G.: The quiet revolution of numerical weather prediction, Nature, 525, 47-55, https://doi.org/https://doi.org/10.1038/nature14956, 2015.

Bihlo, A.: A generative adversarial network approach to (ensemble) weather prediction, arXiv:2006.07718 [physics, stat], 2020.

Brenowitz, N. D., Beucler, T., Pritchard, M., and Bretherton, C. S.: Interpreting and stabilizing machine-learning parametrizations of convection, Journal of the Atmospheric Sciences, 77, 4357-4375, 2020.

Brock, A., Donahue, J., and Simonyan, K.: Large Scale GAN Training for High Fidelity Natural Image Synthesis, in: 7th International Conference on Learning Representations, ICLR 2019, New Orleans, LA, USA, May 6-9, 2019, OpenReview.net, https://openreview.net/ forum?id=B1 xsqj09Fm, 2019.

Canziani, A., Paszke, A., and Culurciello, E.: An analysis of deep neural network models for practical applications, arXiv preprint arXiv:1605.07678, 2016.

Caron, M., Touvron, H., Misra, I., Jégou, H., Mairal, J., Bojanowski, P., and Joulin, A.: Emerging Properties in Self-Supervised Vision

Transformers, in: Proceedings of the IEEE/CVF International Conference on Computer Vision (ICCV), pp. 9650-9660, 2021.

Casaioli, M., Mantovani, R., Proietti Scorzoni, F., Puca, S., Speranza, A., and Tirozzi, B.: Linear and nonlinear post-processing of numerically forecasted surface temperature, Nonlinear Processes in Geophysics, 10, 373-383, https://doi.org/10.5194/npg-10-373-2003, 2003.

Chantry, M., Hatfield, S., Dueben, P., Polichtchouk, I., and Palmer, T.: Machine learning emulation of gravity wave drag in numerical weather forecasting, Journal of Advances in Modeling Earth Systems, 13, e2021MS002 477, 2021.

Chattopadhyay, A., Nabizadeh, E., and Hassanzadeh, P.: Analog forecasting of extreme-causing weather patterns using deep learning, Journal of Advances in Modeling Earth Systems, 12, e2019MS001 958, 2020.

Chepfer, H., Brogniez, H., and Noël, V.: Diurnal variations of cloud and relative humidity profiles across the tropics, Scientific reports, 9 , $1-9,2019$.

Clark, A., Donahue, J., and Simonyan, K.: Adversarial video generation on complex datasets, arXiv preprint arXiv:1907.06571, 2019.

de Bézenac, E., Pajot, A., and Gallinari, P.: Deep learning for physical processes: incorporating prior scientific knowledge, Journal of Statistical Mechanics: Theory and Experiment, 2019, 124 009, https://doi.org/10.1088/1742-5468/ab3195, 2019.

Deng, J., Dong, W., Socher, R., Li, L.-J., Li, K., and Fei-Fei, L.: ImageNet: A Large-Scale Hierarchical Image Database, in: CVPR09, 2009.

Denton, E. and Fergus, R.: Stochastic video generation with a learned prior, arXiv preprint arXiv:1802.07687, 2018.

Dosovitskiy, A., Beyer, L., Kolesnikov, A., Weissenborn, D., Zhai, X., Unterthiner, T., Dehghani, M., Minderer, M., Heigold, G., Gelly,

S., Uszkoreit, J., and Houlsby, N.: An Image is Worth 16x16 Words: Transformers for Image Recognition at Scale, arXiv preprint arXiv:2010.11929, 2020.

Dueben, P. D. and Bauer, P.: Challenges and design choices for global weather and climate models based on machine learning, Geoscientific Model Development, 11, 3999-4009, 2018.

Ebert, F., Finn, C., Lee, A. X., and Levine, S.: Self-supervised visual planning with temporal skip connections, arXiv preprint arXiv:1710.05268, 2017. 
https://doi.org/10.5194/gmd-2021-430

Preprint. Discussion started: 8 March 2022

(c) Author(s) 2022. CC BY 4.0 License.

(c) (i)

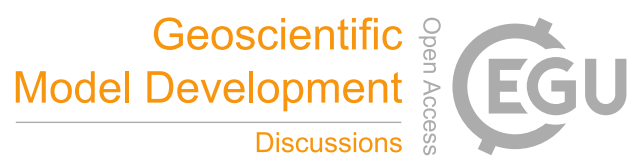

Eccel, E., Ghielmi, L., Granitto, P., Barbiero, R., Grazzini, F., and Cesari, D.: Prediction of minimum temperatures in an alpine region by linear and non-linear post-processing of meteorological models, Nonlinear Processes in Geophysics, 14, 211-222, https://doi.org/10.5194/npg-14-211-2007, 2007.

Efron, B. and Tibshirani, R. J.: An introduction to the bootstrap, CRC press, https://doi.org/10.1201/9780429246593, 1994.

Espeholt, L., Agrawal, S., Sønderby, C., Kumar, M., Heek, J., Bromberg, C., Gazen, C., Hickey, J., Bell, A., and Kalchbrenner, N.: Skillful Twelve Hour Precipitation Forecasts using Large Context Neural Networks, arXiv preprint arXiv:2111.07470, 2021.

Franceschi, J.-Y., Delasalles, E., Chen, M., Lamprier, S., and Gallinari, P.: Stochastic latent residual video prediction, in: International Conference on Machine Learning, pp. 3233-3246, PMLR, 2020.

Ginzburg, A. I., Kostianoy, A. G., and Sheremet, N. A.: Sea surface temperature variability, in: The Black Sea Environment, pp. 255-275, Springer, 2007.

Goodfellow, I., Pouget-Abadie, J., Mirza, M., Xu, B., Warde-Farley, D., Ozair, S., Courville, A., and Bengio, Y.: Generative adversarial nets, in: Advances in neural information processing systems, pp. 2672-2680, 2014.

Grönquist, P., Yao, C., Ben-Nun, T., Dryden, N., Dueben, P., Li, S., and Hoefler, T.: Deep learning for post-processing ensemble weather forecasts, Philosophical Transactions of the Royal Society A, 379, 20200 092, 2021.

650 Guen, V. L. and Thome, N.: Disentangling physical dynamics from unknown factors for unsupervised video prediction, in: Proceedings of the IEEE/CVF Conference on Computer Vision and Pattern Recognition, pp. 11474-11 484, 2020.

Gulrajani, I., Ahmed, F., Arjovsky, M., Dumoulin, V., and Courville, A.: Improved Training of Wasserstein GANs, 2017.

Haiden, T., Janousek, M., Vitart, F., Ben-Bouallegue, Z., Ferranti, L., and Prates, F.: Evaluation of ECMWF forecasts, including the 2021 upgrade, https://doi.org/10.21957/90pgicjk4, 2021.

Han, Y., Zhang, G. J., Huang, X., and Wang, Y.: A moist physics parameterization based on deep learning, Journal of Advances in Modeling Earth Systems, 12, e2020MS002 076, 2020.

Hatfield, S., Chantry, M., Dueben, P., Lopez, P., Geer, A., and Palmer, T.: Building Tangent-Linear and Adjoint Models for Data Assimilation With Neural Networks, Journal of Advances in Modeling Earth Systems, 13, e2021MS002 521, 2021.

Hersbach, H., Bell, B., Berrisford, P., Hirahara, S., Horányi, A., Muñoz-Sabater, J., Nicolas, J., Peubey, C., Radu, R., Schepers, D., et al.: The ERA5 global reanalysis, Quarterly Journal of the Royal Meteorological Society, 146, 1999-2049, 2020.

Isola, P., Zhu, J.-Y., Zhou, T., and Efros, A. A.: Image-to-image translation with conditional adversarial networks, in: Proceedings of the IEEE conference on computer vision and pattern recognition, pp. 1125-1134, 2017.

Jin, B., Hu, Y., Tang, Q., Niu, J., Shi, Z., Han, Y., and Li, X.: Exploring spatial-temporal multi-frequency analysis for high-fidelity and temporal-consistency video prediction, in: Proceedings of the IEEE/CVF Conference on Computer Vision and Pattern Recognition, pp. 4554-4563, 2020.

Jülich Supercomputing Centre: JUWELS: Modular Tier-0/1 Supercomputer at the Jülich Supercomputing Centre, Journal of large-scale research facilities, 5, https://doi.org/10.17815/jlsrf-5-171, 2019.

Karniadakis, G. E., Kevrekidis, I. G., Lu, L., Perdikaris, P., Wang, S., and Yang, L.: Physics-informed machine learning, Nature Reviews Physics, 3, 422-440, 2021.

670 Karpatne, A., Watkins, W., Read, J., and Kumar, V.: Physics-guided neural networks (pgnn): An application in lake temperature modeling, arXiv preprint arXiv:1710.11431, 2017. 
https://doi.org/10.5194/gmd-2021-430

Preprint. Discussion started: 8 March 2022

(c) Author(s) 2022. CC BY 4.0 License.

(c) (i)

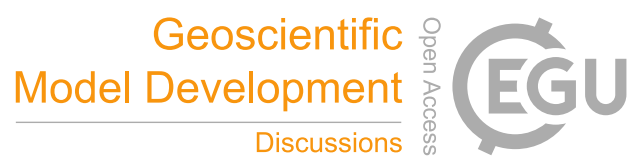

Khain, A., Beheng, K., Heymsfield, A., Korolev, A., Krichak, S., Levin, Z., Pinsky, M., Phillips, V., Prabhakaran, T., Teller, A., et al.: Representation of microphysical processes in cloud-resolving models: Spectral (bin) microphysics versus bulk parameterization, Reviews of Geophysics, 53, 247-322, 2015.

Kim, S., Hong, S., Joh, M., and Song, S.-k.: Deeprain: Convlstm network for precipitation prediction using multichannel radar data, arXiv preprint arXiv:1711.02316, 2017.

Kingma, D. P. and Ba, J.: Adam: A method for stochastic optimization, arXiv preprint arXiv:1412.6980, 2014.

Kingma, D. P. and Welling, M.: Auto-encoding variational bayes, arXiv preprint arXiv:1312.6114, 2013.

Kong, Y. and Fu, Y.: Human action recognition and prediction: A survey, arXiv preprint arXiv:1806.11230, 2018.

Krizhevsky, A., Sutskever, I., and Hinton, G. E.: ImageNet Classification with Deep Convolutional Neural Networks, in: Advances in Neural Information Processing Systems, edited by Pereira, F., Burges, C. J. C., Bottou, L., and Weinberger, K. Q., vol. 25, Curran Associates, Inc., https://proceedings.neurips.cc/paper/2012/file/c399862d3b9d6b76c8436e924a68c45b-Paper.pdf, 2012.

Lee, A. X., Zhang, R., Ebert, F., Abbeel, P., Finn, C., and Levine, S.: Stochastic adversarial video prediction, arXiv preprint arXiv:1804.01523, 2018.

Leinonen, J., Nerini, D., and Berne, A.: Stochastic super-resolution for downscaling time-evolving atmospheric fields with a generative adversarial network, IEEE Transactions on Geoscience and Remote Sensing, https://doi.org/10.1109/TGRS.2020.3032790, 2020.

Lezama Valdes, L.-M., Katurji, M., and Meyer, H.: A Machine Learning Based Downscaling Approach to Produce High Spatio-Temporal Resolution Land Surface Temperature of the Antarctic Dry Valleys from MODIS Data, Remote Sensing, 13, 4673, 2021.

Liu, H.-B. and Lee, I.: MPL-GAN: Toward Realistic Meteorological Predictive Learning Using Conditional GAN, IEEE Access, 8, 93 179_ 93 186, 2020.

Liu, W., Luo, W., Lian, D., and Gao, S.: Future frame prediction for anomaly detection-a new baseline, in: Proceedings of the IEEE conference on computer vision and pattern recognition, pp. 6536-6545, 2018.

Lorenz, E. N.: The predictability of a flow which possesses many scales of motion, Tellus, 21, 289-307, https://doi.org/10.3402/tellusa.v21i3.10086, 1969.

Mathieu, M., Couprie, C., and LeCun, Y.: Deep multi-scale video prediction beyond mean square error, arXiv preprint arXiv:1511.05440, 2015.

McGovern, A., Elmore, K. L., Gagne, D. J., Haupt, S. E., Karstens, C. D., Lagerquist, R., Smith, T., and Williams, J. K.: Using artificial intelligence to improve real-time decision-making for high-impact weather, Bulletin of the American Meteorological Society, 98, 20732090, 2017.

700 Murphy, A. H. and Winkler, R. L.: A general framework for forecast verification, Monthly weather review, 115, 1330-1338, 1987.

Oliu, M., Selva, J., and Escalera, S.: Folded recurrent neural networks for future video prediction, in: Proceedings of the European Conference on Computer Vision (ECCV), pp. 716-731, 2018.

Oprea, S., Martinez-Gonzalez, P., Garcia-Garcia, A., Castro-Vargas, J. A., Orts-Escolano, S., Garcia-Rodriguez, J., and Argyros, A.: A review on deep learning techniques for video prediction, IEEE Transactions on Pattern Analysis and Machine Intelligence, 2020.

Orlanski, I.: A rational subdivision of scales for atmospheric processes, Bulletin of the American Meteorological Society, pp. 527-530, https://doi.org/https://doi.org/10.1175/1520-0477-56.5.527, 1975.

Patraucean, V., Handa, A., and Cipolla, R.: Spatio-temporal video autoencoder with differentiable memory, arXiv preprint arXiv:1511.06309, 2015. 
https://doi.org/10.5194/gmd-2021-430

Preprint. Discussion started: 8 March 2022

(c) Author(s) 2022. CC BY 4.0 License.

(c) (i)

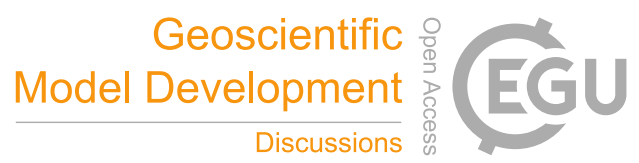

Prudden, R., Adams, S., Kangin, D., Robinson, N., Ravuri, S., Mohamed, S., and Arribas, A.: A review of radar-based nowcasting of precipitation and applicable machine learning techniques, arXiv preprint arXiv:2005.04988, 2020.

Qi, M., Wang, Y., Li, A., and Luo, J.: STC-GAN: Spatio-temporally coupled generative adversarial networks for predictive scene parsing, IEEE Transactions on Image Processing, 29, 5420-5430, 2020.

Racah, E., Beckham, C., Maharaj, T., Prabhat, and Pal, C. J.: Semi-Supervised Detection of Extreme Weather Events in Large Climate Datasets, CoRR, abs/1612.02095, http://arxiv.org/abs/1612.02095, 2016.

715 Rao, Q. and Frtunikj, J.: Deep learning for self-driving cars: Chances and challenges, in: Proceedings of the 1st International Workshop on Software Engineering for AI in Autonomous Systems, pp. 35-38, 2018.

Rasp, S. and Lerch, S.: Neural networks for postprocessing ensemble weather forecasts, Monthly Weather Review, 146, 3885-3900, https://doi.org/10.1175/MWR-D-18-0187.1, 2018.

Rasp, S. and Thuerey, N.: Data-Driven Medium-Range Weather Prediction With a Resnet Pretrained on Climate Simulations: A New Model for WeatherBench, Journal of Advances in Modeling Earth Systems, 13, https://doi.org/10.1029/2020MS002405, 2021.

Rasp, S., Dueben, P. D., Scher, S., Weyn, J. A., Mouatadid, S., and Thuerey, N.: WeatherBench: a benchmark data set for data-driven weather forecasting, Journal of Advances in Modeling Earth Systems, 12, e2020MS002 203, 2020.

Ravuri, S., Lenc, K., Willson, M., Kangin, D., Lam, R., Mirowski, P., Fitzsimons, M., Athanassiadou, M., Kashem, S., Madge, S., Prudden, R., Mandhane, A., Clark, A., Brock, A., Simonyan, K., Hadsell, R., Robinson, N., Clancy, E., Arribas, A., and Mohamed, S.: Skilful precipitation nowcasting using deep generative models of radar, Nature, 597, 672-677, https://doi.org/10.1038/s41586-021-03854-z, 2021.

Reichstein, M., Camps-Valls, G., Stevens, B., Jung, M., Denzler, J., Carvalhais, N., et al.: Deep learning and process understanding for data-driven Earth system science, Nature, 566, 195-204, 2019.

Reyniers, M.: Quantitative precipitation forecasts based on radar observations: Principles, algorithms and operational systems, Institut Royal Météorologique de Belgique Brussel, Belgium, 2008.

Scher, S.: Toward data-driven weather and climate forecasting: Approximating a simple general circulation model with deep learning, Geophysical Research Letters, 45, 12-616, 2018.

Schultz, M. G., Betancourt, C., Gong, B., Kleinert, F., Langguth, M., Leufen, L. H., Mozaffari, A., and Stadtler, S.: Can deep learning beat numerical weather prediction?, Philosophical Transactions of the Royal Society A: Mathematical, Physical and Engineering Sciences, 379, https://doi.org/10.1098/rsta.2020.0097, 2021.

Sha, Y., Gagne II, D. J., West, G., and Stull, R.: Deep-learning-based gridded downscaling of surface meteorological variables in complex terrain. Part I: Daily maximum and minimum 2-m temperature, Journal of Applied Meteorology and Climatology, 59, 2057-2073, 2020.

Sønderby, C. K., Espeholt, L., Heek, J., Dehghani, M., Oliver, A., Salimans, T., Agrawal, S., Hickey, J., and Kalchbrenner, N.: Metnet: A neural weather model for precipitation forecasting, arXiv preprint arXiv:2003.12140, 2020.

Sun, J., Xue, M., Wilson, J. W., Zawadzki, I., Ballard, S. P., Onvlee-Hooimeyer, J., Joe, P., Barker, D. M., Li, P.-W., Golding, B., et al.: Use of NWP for nowcasting convective precipitation: Recent progress and challenges, Bulletin of the American Meteorological Society, 95, 409-426, 2014.

Sun, J., Xie, J., Hu, J.-F., Lin, Z., Lai, J., Zeng, W., and Zheng, W.-S.: Predicting future instance segmentation with contextual pyramid convlstms, in: Proceedings of the 27th acm international conference on multimedia, pp. 2043-2051, 2019.

Tarek, M., Brissette, F. P., and Arsenault, R.: Evaluation of the ERA5 reanalysis as a potential reference dataset for hydrological modelling over North America, Hydrology and Earth System Sciences, 24, 2527-2544, 2020. 
https://doi.org/10.5194/gmd-2021-430

Preprint. Discussion started: 8 March 2022

(c) Author(s) 2022. CC BY 4.0 License.

(c) (i)

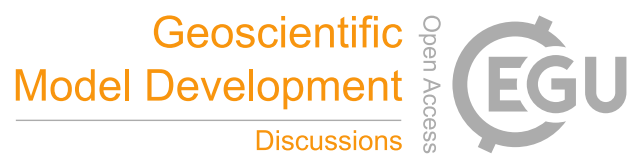

Vannitsem, S., Bremnes, J. B., Demaeyer, J., Evans, G. R., Flowerdew, J., Hemri, S., Lerch, S., Roberts, N., Theis, S., Atencia, A., et al.: Statistical Postprocessing for Weather Forecasts: Review, Challenges, and Avenues in a Big Data World, Bulletin of the American Meteorological Society, 102, E681-E699, 2021.

Villegas, R., Yang, J., Hong, S., Lin, X., and Lee, H.: Decomposing motion and content for natural video sequence prediction, arXiv preprint arXiv:1706.08033, 2017.

von Engeln, A. and Teixeira, J.: A planetary boundary layer height climatology derived from ECMWF reanalysis data, Journal of Climate, 26, 6575-6590, 2013.

Wang, Y., Long, M., Wang, J., Gao, Z., and Philip, S. Y.: Predrnn: Recurrent neural networks for predictive learning using spatiotemporal 1stms, in: Advances in Neural Information Processing Systems, pp. 879-888, 2017.

Wang, Y., Jiang, L., Yang, M.-H., Li, L.-J., Long, M., and Fei-Fei, L.: Eidetic 3d 1stm: A model for video prediction and beyond, in: International conference on learning representations, 2018.

Wang, Y., Wu, H., Zhang, J., Gao, Z., Wang, J., Yu, P. S., and Long, M.: PredRNN: A Recurrent Neural Network for Spatiotemporal Predictive Learning, arXiv preprint arXiv:2103.09504, 2021.

Wang, Z., Bovik, A. C., Sheikh, H. R., and Simoncelli, E. P.: Image quality assessment: from error visibility to structural similarity, IEEE transactions on image processing, 13, 600-612, 2004.

Weyn, J. A., Durran, D. R., and Caruana, R.: Can machines learn to predict weather? Using deep learning to predict gridded 500-hPa geopotential height from historical weather data, Journal of Advances in Modeling Earth Systems, 11, 2680-2693, 2019.

Weyn, J. A., Durran, D. R., and Caruana, R.: Improving data-driven global weather prediction using deep convolutional neural networks on a cubed sphere, Journal of Advances in Modeling Earth Systems, 12, e2020MS002 109, 2020.

Wilks, D. S.: Statistical methods in the atmospheric sciences, vol. 100, Academic press, 2011.

Wilson, J. W., Feng, Y., Chen, M., and Roberts, R. D.: Nowcasting challenges during the Beijing Olympics: Successes, failures, and implications for future nowcasting systems, Weather and Forecasting, 25, 1691-1714, 2010.

Xingjian, S., Chen, Z., Wang, H., Yeung, D.-Y., Wong, W.-K., and Woo, W.-c.: Convolutional LSTM network: A machine learning approach for precipitation nowcasting, in: Advances in neural information processing systems, pp. 802-810, 2015.

770 Yan, W., Zhang, Y., Abbeel, P., and Srinivas, A.: VideoGPT: Video Generation using VQ-VAE and Transformers, arXiv preprint arXiv:2104.10157, 2021.

Zaengl, G., Reinert, D., Rípodas, P., and Baldauf, M.: The ICON (ICOsahedral Non-hydrostatic) modelling framework of DWD and MPI-M: Description of the non-hydrostatic dynamical core, Quarterly Journal of the Royal Meteorological Society, 141, 563-579, 2015.

Ziyin, L., Hartwig, T., and Ueda, M.: Neural networks fail to learn periodic functions and how to fix it, arXiv preprint arXiv:2006.08195, 2020. 